\title{
Model Selection in the Presence of Incidental Parameters
}

\author{
Yoonseok Lee* and Peter C.B. Phillips ${ }^{\dagger}$
}

April 2014

\begin{abstract}
This paper considers model selection in panels where incidental parameters are present. Primary interest centers on selecting a model that best approximates the underlying structure involving parameters that are common within the panel. It is well known that conventional model selection procedures are often inconsistent in panel models and this can be so even without nuisance parameters. Modifications are then needed to achieve consistency. New model selection information criteria are developed here that use either the Kullback-Leibler information criterion based on the profile likelihood or the Bayes factor based on the integrated likelihood with a bias-reducing prior. These model selection criteria impose heavier penalties than those associated with standard information criteria such as AIC and BIC. The additional penalty, which is data-dependent, properly reflects the model complexity arising from the presence of incidental parameters. A particular example is studied in detail involving lag order selection in dynamic panel models with fixed effects. The new criteria are shown to control for over/under-selection probabilities in these models and lead to consistent order selection criteria.
\end{abstract}

Keywords: (Adaptive) model selection, incidental parameters, profile likelihood, KullbackLeibler information, integrated likelihood, bias-reducing prior, fixed effects, lag order.

JEL Classifications: C23, C52

*Syracuse University. Address: Department of Economics and Center for Policy Research, Syracuse University, 426 Eggers Hall, Syracuse, NY 13244-1020. E-mail: ylee41@maxwell.syr.edu.

${ }^{\dagger}$ Yale University, University of Auckland, University of Southampton and Singapore Management University. Address: Department of Economics, Yale University, Box 208281, New Haven, CT 06520-8281. E-mail: peter.phillips@yale.edu. 


\section{Introduction}

As datasets grow richer, more sophisticated models are being used in empirical econometric work, including semiparametric models, large dimensional parametric models, and panel systems with manifold heterogenous effects that lead to a proliferation of nuisance parameters. Good model selection procedures are an important element in empirical work to avoid bias, to help in validating inference, and to assist in ensuring sound policy implications. They are particularly important in more sophisticated systems where multi-index asymptotics and high dimensional nuisance parameters can affect the properties of estimators, inference and model selection.

Some of these panel modeling issues were considered in the pioneering work by Anderson and Hsiao (1981), which examined the use of multi-index asymptotics, dynamic panel estimation inconsistency, and the possible use of instrumental variable (IV) methods to avoid inconsistencies in dynamic panel regression with short wide panels 11 Following that paper, there was a flowering of research on dynamic panel modeling, efficient IV estimation techniques and semiparametric methods, to all of which Cheng Hsiao has made significant contributions. Much of this work is overviewed in Hsiao (2003).

One topic that is still relatively unexplored in this field is model selection in dynamic panels. Specification tests and information-criteria provide two standard approaches to model selection and are available for use in dynamic panels. The specification test approach requires an ad hoc null, a set of alternative models, and a test sequence to evaluate the alternatives. On the other hand, the model selection approach considers all the candidate models jointly and chooses one that optimizes an information criterion. Examples include the Akaike information criterion (AIC), Bayesian information criterion (BIC), posterior information criterion (PIC), Hannan-Quinn (HQ) criterion, the Mellows' $C_{p}$ criterion, bootstrap criteria and crossvalidation approaches. See, for example, Claeskens and Hjort (2008) for further details of these methods.

${ }^{1}$ Phillips (2014) and Phillips and Han (2014) provide some updated asymptotic results on the Anderson and Hsiao IV estimators. 
An important assumption in most model selection approaches is that the number of parameters in each candidate model is finite or at most grows slowly compared to the sample size. For example, Stone (1979) showed that consistency of the standard BIC order selector breaks down when the number of parameters in the candidate model diverges with the sample size $2^{2}$ In many cases, large dimensional parameter spaces arise from the proliferation of nuisance parameters which, though they are not of primary interest, are required for specifying heterogeneity or for handling omitted variables. The present paper examines why standard model selection criteria perform poorly for such cases and proposes modified selection criteria that are effective when the candidate models have nuisance parameters whose dimension grows with the sample size, analogous to incidental parameters (Neyman and Scott (1948)).

In particular, we study the specification of panel data models in which the focus of interest is a subset of the parameters. We consider panel observations $z_{i, t}$ for $i=1, \cdots, n$ and $t=1, \cdots, T$, whose unknown density (i.e., the model) is approximated by a parametric family $f\left(z ; \psi, \lambda_{i}\right)$ that does not need to include the true model. The parameter of interest is $\psi$, which is common across $i$, and the nuisance parameters are given by $\lambda_{1}, \cdots, \lambda_{n}$, whose number increases at the same rate of the sample size. Common examples of $\lambda_{i}$ are unobserved heterogeneity (e.g., individual fixed effects) and heteroskedastic variances. The main objective is to choose the model that fits best the data generating process when only a subset of the parameters is of central interest. Such an approach is reasonable when we are interested in selecting the structure of the model in $\psi$, while assuming the parameter space of $\lambda_{i}$ is common across the candidate models. A similar approach can be found in Claeskens and Hjort (2003) in the context of cross section models with finite-dimensional nuisance parameters, though they consider the case with nested models via local misspecification. In comparison, we allow for infinite-dimensional nuisance parameters as well as nonnested cases.

Two different approaches are used to handle incidental parameters and to obtain new model selection criteria. One method applies profiling to the Kullback-Leibler information

\footnotetext{
${ }^{2}$ This limitation in standard criteria is now well understood and several approaches have been proposed for model selection in large dimensional models, particularly in the Bayesian framework. Examples are Berger et al. (2003) and Chakrabarti and Ghosh (2006), who analyze the use of the Laplace approximation in largedimensional exponential families to compute the Bayes factor and achieve a consistent selector.
} 
criterion (KLIC). It is shown that the profile KLIC can be approximated by the standard KLIC based on the profile likelihoods provided that a proper modification term is imposed. This result corresponds to the fact that the profile likelihood does not share the standard properties of the genuine likelihood function (e.g., the score has nonzero expectation or the information identity is violated), which therefore needs appropriate modification (e.g., Sartori $(2003))$. It turns out that the new information criterion requires a heavier penalty than that of standard information criteria such as AIC so that the degrees of freedom in the model are properly counted. However, the penalty is different from the total number of parameters (i.e., $\left.\operatorname{dim}(\psi)+n \operatorname{dim}\left(\lambda_{i}\right)\right)$. The additional penalty depends on a proper model complexity measure (e.g., Rissanen (1986), Bozdogan (2000), Hodges and Sargent (2001)) that reflects the level of difficulty of estimation. The penalty term is data-dependent, so the new model selection rule is adaptive. As a second approach, we develop a Bayesian model selection criterion that is based on the Bayes factor, in which the posterior is obtained using the integrated likelihoods. These two approaches - one based on the profile likelihood and the other based on the integrated likelihood - are closely related, as in the standard AIC and BIC, provided that a proper prior for the incidental parameter is used in performing the integration. In the pseudo-likelihood setup, we obtain the prior so that the integrated likelihood is close to the genuine likelihood (e.g., the robust prior of Arellano and Bonhomme (2009)) and that depends on the data in general.

The majority of panel studies focus on modifying the profile or integrated likelihood as a means of bias reduction in maximum likelihood estimation, which presume that the parametric models considered are correctly specified (e.g., Hahn and Kuersteiner (2002, 2011); Hahn and Newey (2004); Arellano and Hahn (2006, 2007); Lee (2006, 2012, 2014); Bester and Hansen (2009) ). However, as discussed in Lee (2006, 2012), if the model is not correctly specified, effort to reduce bias stemming from incidental parameters may exacerbate bias. Hence, correct model specification is very important, particularly for dynamic or nonlinear panel models where bias occurs naturally in estimation. Correct model specification should ideally precede the use of bias correction or bias reduction procedures. The focus of the 
present paper is on mechanisms to address the specification problem.

The remainder of the paper is organized as follows. Section 2 summarizes the incidental parameter problem in the quasi maximum likelihood setup. The modified profile likelihood and bias reduction in panel models are also discussed. Section 3 develops an AIC-type information criterion based on the profile likelihood. A profile KLIC is introduced that is general enough to be applied in heterogenous panel data models. Section 4 obtains a BIC-type information criterion based on the integrated likelihood and explores connections between AIC-type and BIC-type criteria by developing a robust prior. In Section 5, the methodology is mobilized in the particular example of lag order selection for dynamic panels. This Section also reports simulations that examine the statistical performance properties of the procedures. Section 6 concludes. Proofs are given in the Appendix.

\section{Incidental Parameter Problems in QMLE}

\subsection{Misspecified models}

We consider panel observations $\left\{z_{i, t}\right\}$ for $i=1,2, \cdots, n$ and $t=1,2, \cdots, T$, which have an unknown distribution $G_{i}(z)$ with probability density $g_{i}(z)$. The components $z_{i, t}$ are allowed to have heterogenous distributions across $i$ but are cross-section independent $t^{3}$ On the other hand, $z_{i, t}$ may be serially correlated over $t$ but is assumed to be strictly stationary so that the marginal distribution of $z_{i, t}$ is invariant in $t$. $T$ could vary over $i$ (i.e., $T_{i} \neq T_{j}$ ) but we assume $T_{i}=T$ for all $i$ for simplicity in what follows. Since $g_{i}(z)$ is unknown a priori, we consider a parametric family of densities $\left\{f\left(z ; \theta_{i}\right): \theta_{i} \in \Theta\right\}$ for each $i$, which does not necessarily contain $g_{i}(z)$. We assume that $f\left(z ; \theta_{i}\right)$ is continuous (and smooth enough as needed) in $\theta_{i}$ for every $z \in \mathcal{Z}$, the usual regularity conditions for $f\left(z ; \theta_{i}\right)$ hold (e.g., Severini (2000), Chapter 4), and that the parameters are all well identified. Note that the heterogeneity of the marginal distribution is solely controlled by the heterogenous parameter $\theta_{i}$. We decompose the parameter vector as $\theta_{i}=\left(\psi^{\prime}, \lambda_{i}\right)^{\prime}$, where $\psi \in \Psi \subset \mathbb{R}^{r}$ is the main parameter of interest

\footnotetext{
${ }^{3}$ Mild cross-section dependence could be permitted under some commonly used frameworks such as a factor structure as long as the LLN and CLT can be extended. Cross section independence is assumed here for expositional simplicity.
} 
common to all $i$, whereas the $\lambda_{i} \in \Lambda \subset \mathbb{R}$ are individual nuisance parameter that are specific to $i$. Panel models with heterogenous parameters, such as fixed individual effects, (conditional) heteroskedasticity, or heterogenous slope coefficients, are good examples of $f\left(\cdot ; \psi, \lambda_{i}\right)$. We may consider multidimensional $\lambda_{i}$ (e.g., Arellano and Hahn (2006)) but focus on the scalar case for expositional simplicity.

We denote the marginal (pseudo-)likelihood of $z_{i, t}$ as $4^{4}$

$$
f_{i t}\left(\psi, \lambda_{i}\right)=f\left(z_{i, t} ; \psi, \lambda_{i}\right)
$$

which leads to the expression for the scaled individual log-likelihood function given by

$$
\ell_{i}\left(\psi, \lambda_{i}\right)=\frac{1}{T} \sum_{t=1}^{T} \log f_{i t}\left(\psi, \lambda_{i}\right)
$$

We assume the following conditions as in White (1982). Though $z_{i, t}$ may be serially correlated, we assume the process is stationary mixing and the serial dependence is sufficiently weak for the LLN and CLT to hold (e.g., Hahn and Kuersteiner (2011), Lee (2014)). We let a measurable Euclidean space $\mathcal{Z}$ be the support of $z_{i, t}$.

Assumption 1 (i) $z_{i, t}$ is independent over $i$ and (for each $i$ ) strictly stationary strong mixing over $t$ with marginal distribution $G_{i}$ on $\mathcal{Z}$ and measurable Radon-Nikodym density $g_{i}=d G_{i} / d \nu$. (ii) For each $i, f\left(z ; \theta_{i}\right)$ is the Radon-Nikodym density of the distribution $F\left(z ; \theta_{i}\right)$, where $f\left(z ; \theta_{i}\right)$ is measurable in $z$ for every $\theta_{i} \in \Theta=\Psi \times \Lambda$, a compact subset of $\mathbb{R}^{r+1}$ and twice continuously differentiable in $\theta_{i}$ for every $z \in \mathcal{Z}$. (iii) It can be decomposed as $\theta_{i}=\left(\psi^{\prime}, \lambda_{i}\right)^{\prime}$, where $\lambda_{i}$ is related to the $i$-th observation only.

Since we are mainly interested in $\psi$, we first maximize out the nuisance parameter $\lambda_{i}$ to

\footnotetext{
${ }^{4}$ When we consider dynamic models, $f\left(z_{i, t} ; \psi, \lambda_{i}\right)$ is understood as a conditional density given the lagged observations. For example, with $z_{i, t}=\left(y_{i, t}, y_{i, t-1}, \cdots, y_{i, t-p}\right)$ for some $p \geq 1$, we define $f\left(z_{i, t} ; \psi, \lambda_{i}\right)=$ $f\left(y_{i, t} \mid y_{i, t-1}, \cdots, y_{i, t-p} ; \psi, \lambda_{i}\right)$.
} 
define the profile likelihood of $\psi$ as

$$
f_{i t}^{P}(\psi)=f\left(z_{i, t} ; \psi, \widehat{\lambda}_{i}(\psi)\right) \text { for each } i
$$

where

$$
\widehat{\lambda}_{i}(\psi)=\arg \max _{\lambda_{i} \in \Lambda} \ell_{i}\left(\psi, \lambda_{i}\right)
$$

is the quasi maximum likelihood estimator (QMLE) of $\lambda_{i}$ keeping $\psi$ fixed. Note that (3) is possible since the nuisance parameter is separable in $i$. By separability, furthermore, we can consider the standard asymptotic results for $\widehat{\lambda}_{i}(\psi)$ in powers of $T$. The quasi maximum profile likelihood estimator of $\psi$ is then obtained as

$$
\widehat{\psi}=\arg \max _{\psi \in \Psi} \frac{1}{n} \sum_{i=1}^{n} \ell_{i}^{P}(\psi), \text { where } \ell_{i}^{P}(\psi)=\frac{1}{T} \sum_{t=1}^{T} \log f_{i t}^{P}(\psi)
$$

which indeed corresponds to the QMLE of $\psi$ because the maximum is obtained in two successive steps rather than simultaneously. The existence of $\widehat{\psi}$ follows from Assumption 1 as in White (1982). When $T$ is small, however, $f_{i t}^{P}(\psi)$ does not behave like the standard likelihood function due to the sampling variability of the estimator $\widehat{\lambda}_{i}(\psi)$. For example, the expected score of the profile likelihood is nonzero and the standard information identity does not hold even when the true density is nested in $\left\{f\left(\cdot ; \psi, \lambda_{i}\right)\right\}$. The intuitive explanation is that the profile likelihood is itself a biased estimate of the original likelihood. Modification of the profile likelihoods in the form of

$$
\ell_{i}^{M}(\psi)=\ell_{i}^{P}(\psi)-\frac{1}{T} M_{i}(\psi)=\frac{1}{T} \sum_{t=1}^{T} \log f_{i t}^{M}(\psi)
$$

is widely studied, where

$$
\log f_{i t}^{M}(\psi)=\log f_{i t}^{P}(\psi)-\frac{1}{T} M_{i}(\psi)
$$

Such modification makes the modified profile likelihood $f_{i t}^{M}(\psi)$ behave more like a genuine 
likelihood function (e.g., Barndorff-Nielsen (1983)). The modification term $M_{i}(\psi)$ is $O_{p}(1)$ and $M_{i}(\psi) / T$ corrects the leading $O_{p}\left(T^{-1}\right)$ sampling bias from $\widehat{\lambda}_{i}(\psi)$ so that it renders the expected score of the modified profile likelihood closer to zero even for small T. A bias-reduced estimator for $\psi$ can therefore be obtained by maximizing the modified profile likelihood (i.e., the quasi maximum modified profile likelihood estimation) as

$$
\widetilde{\psi}=\arg \max _{\psi \in \Psi} \frac{1}{n} \sum_{i=1}^{n} \ell_{i}^{M}(\psi)
$$

Further discussion of the maximum modified profile likelihood estimator can be found in Barndorff-Nielsen (1983), Severini (1998, 2000) and Sartori (2003) among others, particularly regarding appropriate choices of the modification term $M_{i}(\psi)$. Closely related works consider the adjusted profile likelihood (e.g., McCullagh and Tibshirani (1990), DiCiccio et al. (1996)) and the conditional profile likelihood (e.g., Cox and Reid (1987)).

\subsection{Incidental parameter problem}

From standard QMLE theory we can show that the QML estimator (or the quasi maximum

profile likelihood estimator) $\widehat{\psi}$ in $(4)$ is a consistent estimator for a nonrandom vector $\psi_{T}$ for fixed $T$, where

$$
\psi_{T}=\arg \max _{\psi \in \Psi} \lim _{n \rightarrow \infty} \frac{1}{n} \sum_{i=1}^{n} \mathbb{E}_{G_{i}}\left[\frac{1}{T} \sum_{t=1}^{T} \log f\left(z_{i, t} ; \psi, \widehat{\lambda}_{i}(\psi)\right)\right] .
$$

Note that $\widehat{\lambda}_{i}(\psi)$ in $(3)$ is a function of $T$. We denote by $\mathbb{E}_{G_{i}}[\cdot]=\int[\cdot] d G_{i}$ the expectation taken with respect to the true distribution $G_{i}$ for each $i . \psi_{T}$ can be rewritten as

$$
\psi_{T}=\arg \min _{\psi \in \Psi} \lim _{n \rightarrow \infty} \frac{1}{n T} \sum_{i=1}^{n} \sum_{t=1}^{T} D\left(g_{i} \| f_{i t}\left(\psi, \widehat{\lambda}_{i}(\psi)\right)\right)
$$

where for each $i$

$$
D\left(g_{i} \| f_{i t}\left(\psi, \widehat{\lambda}_{i}(\psi)\right)\right)=\mathbb{E}_{G_{i}}\left[\log \left(\frac{g_{i}\left(z_{i, t}\right)}{f\left(z_{i, t} ; \psi, \widehat{\lambda}_{i}(\psi)\right)}\right)\right]
$$


is the Kullback-Leibler divergence (or the Kullback-Leibler information criterion - KLIC) of the true marginal density $g_{i}(\cdot)$ relative to $\left.f\left(\cdot ; \psi, \widehat{\lambda}_{i}(\psi)\right)\right)$, which is well defined by the conditions below ${ }^{5}$ Using stationarity, we further let ${ }^{6}$

$$
\lambda_{i}(\psi)=\arg \min _{\lambda_{i} \in \Lambda} D\left(g_{i} \| f_{i t}\left(\psi, \lambda_{i}\right)\right)
$$

for each $i$ and

$$
\begin{aligned}
\psi_{0} & =\arg \max _{\psi \in \Psi} \lim _{n \rightarrow \infty} \frac{1}{n} \sum_{i=1}^{n} \mathbb{E}_{G_{i}}\left[\log f\left(z_{i, t} ; \psi, \lambda_{i}(\psi)\right)\right] \\
& =\arg \min _{\psi \in \Psi} \lim _{n \rightarrow \infty} \frac{1}{n} \sum_{i=1}^{n} D\left(g_{i} \| f_{i t}\left(\psi, \lambda_{i}(\psi)\right)\right) .
\end{aligned}
$$

The KLIC minimizers $\psi_{0}$ and $\lambda_{0}=\left(\lambda_{10}, \cdots, \lambda_{n 0}\right)^{\prime}$ are obtained from (9) and $\lambda_{i 0}=\lambda_{i}\left(\psi_{0}\right)$ for each $i$.

Assumption 2 For each $i$, (i) both $g_{i}(z)$ and $f\left(z ; \theta_{i}\right)$ are bounded away from zero for all $z$ in $\mathcal{Z}$ and $\theta_{i}$ in $\Theta$; (ii) $\mathbb{E}_{G_{i}}\left[\log g_{i}\left(z_{i, t}\right)\right]$ exists and $\lim _{n \rightarrow \infty} n^{-1} \sum_{i=1}^{n} \mathbb{E}_{G_{i}}\left[\log g_{i}\left(z_{i, t}\right)\right]<$ $\infty$; (iii) $\partial \log f\left(z ; \theta_{i}\right) / \partial \theta_{i[j]}$ for $j=1, \cdots, r+1$ are measurable functions of $z$ for each $\theta_{i}$ in $\Theta$ and continuously differentiable with respect to $G_{i}$ for all $z$ in $\mathcal{Z}$ and $\theta_{i}$ in $\Theta$, where $\theta_{i[j]}$ denotes the $j$ th element of $\theta_{i} ;(i v)\left|\log f\left(z ; \theta_{i}\right)\right|,\left|\partial^{2} \log f\left(z ; \theta_{i}\right) / \partial \theta_{i[j]} \partial \theta_{i[k]}\right|$ and $\left|\partial \log f\left(z ; \theta_{i}\right) / \partial \theta_{i[j]} \cdot \partial \log f\left(z ; \theta_{i}\right) / \partial \theta_{i[k]}\right|$ are all dominated by functions integrable with respect to $G_{i}$ for all $z$ in $\mathcal{Z}$ and $\theta_{i}$ in $\Theta$ for $j, k=1, \cdots, r+1$, where each dominating function (say $\varsigma(\cdot)$ ) satisfies $\lim _{n \rightarrow \infty} n^{-1} \sum_{i=1}^{n} \mathbb{E}_{G_{i}}\left[\varsigma\left(z_{i, t}\right)\right]<\infty ;(v) \mathbb{E}_{G_{i}}\left[\partial^{2} \log f\left(z_{i, t} ; \theta_{i 0}\right) / \partial \theta_{i} \partial \theta_{i}^{\prime}\right]$ is negative definite and so is $\lim _{n \rightarrow \infty} n^{-1} \sum_{i=1}^{n} \mathbb{E}_{G_{i}}\left[\partial^{2} \log f\left(z_{i, t} ; \theta_{i 0}\right) / \partial \theta_{i} \partial \theta_{i}^{\prime}\right]<\infty$, where $\theta_{i 0}=$ $\left(\psi_{0}^{\prime}, \lambda_{i 0}\right)^{\prime} ;$ (vi) $\mathcal{I}_{i}=\mathbb{E}_{G_{i}}\left[\partial \log f\left(z_{i, t} ; \theta_{i 0}\right) / \partial \theta_{i} \cdot \partial \log f\left(z_{i, t} ; \theta_{i 0}\right) / \partial \theta_{i}^{\prime}\right]$ is nonsingular and so is

\footnotetext{
${ }^{5}$ We may interpret the objective function in 77 as the average KLIC of $g_{i}\left(z_{i, t}\right)$ relative to the scaled individual parametric profile likelihood $\bar{f}_{i}\left(\psi, \widehat{\lambda}_{i}(\psi)\right)=\exp \left[T^{-1} \sum_{t=1}^{T} \log f\left(z_{i, t} ; \psi, \widehat{\lambda}_{i}(\psi)\right)\right]$ since

$$
\left.\frac{1}{T} \sum_{t=1}^{T} D\left(g_{i} \| f_{i t}\left(\psi, \widehat{\lambda}_{i}(\psi)\right)\right)\right)=\mathbb{E}_{G_{i}}\left[\log g_{i}\left(z_{i, t}\right)-\frac{1}{T} \sum_{t=1}^{T} \log f\left(z_{i, t} ; \psi, \widehat{\lambda}_{i}(\psi)\right)\right]=D\left(g_{i} \| \bar{f}_{i}\left(\psi, \widehat{\lambda}_{i}(\psi)\right)\right)
$$

by stationarity.

${ }^{6} \lambda_{i}(\psi)$ is normally referred to as the least favorable curve.
} 
$\lim _{n \rightarrow \infty} n^{-1} \sum_{i=1}^{n} \mathcal{I}_{i}<\infty$; (vii) $\left(\psi_{0}^{\prime}, \lambda_{0}^{\prime}\right)^{\prime} \in \Psi \times \Lambda^{n}$ is the unique solution from (8) and (9), where $\left(\psi_{0}^{\prime}, \lambda_{0}^{\prime}\right)^{\prime}$ lies in the interior of the support.

From White (1982) under Assumptions 1 and 2, we have that $\widehat{\psi}=\psi_{T}+o_{p}(1)$ as $n \rightarrow \infty$ with fixed $T$. When the dimension of the nuisance parameters $\lambda=\left(\lambda_{1}, \cdots, \lambda_{n}\right)^{\prime}$ is substantial relative to the sample size (e.g., when $T$ is small), however, $\psi_{T}$ is usually different from the standard KLIC minimizer $\psi_{0}$ in (9). This inconsistency is a manifestation of the incidental parameter problem (e.g., Neyman and Scott (1948)) in the context of the QMLE. In general, it can often be shown that (e.g., Arellano and Hahn (2007), Bester and Hansen (2009), Hahn and Kuersteiner (2011))

$$
\psi_{T}-\psi_{0}=\frac{\Upsilon}{T}+O\left(\frac{1}{T^{2}}\right)
$$

where $\Upsilon / T$ represents bias of $O\left(T^{-1}\right)$, and when $n, T \rightarrow \infty$ with $n / T \rightarrow \kappa \in(0, \infty)$, we have

$$
\sqrt{n T}\left(\widehat{\psi}-\psi_{0}\right)=\sqrt{n T}\left(\widehat{\psi}-\psi_{T}\right)+\sqrt{\frac{n}{T}} \Upsilon+O_{p}\left(\sqrt{\frac{n}{T^{3}}}\right) \rightarrow{ }_{d} \mathcal{N}\left(\sqrt{\kappa} \Upsilon, \Omega_{\psi}\right)
$$

for some positive definite matrix $\Omega_{\psi}$. The main source of this bias is that $\widehat{\lambda}_{i}(\psi)$ in $(3)$ is still random and thus is not the same as $\lambda_{i}(\psi)$ in $(8)$. The estimation error of $\widehat{\lambda}_{i}(\psi)$ with finite $T$ is not negligible even when $n \rightarrow \infty$, and the expectation of the profile score is no longer zero for each $i$ even under sufficient regularity conditions.

More precisely, for each $i$, we define the (pseudo-)information matrix as

$$
\mathcal{I}_{i}=\mathbb{E}_{G_{i}}\left[\frac{\partial \log f_{i t}\left(\psi_{0}, \lambda_{i 0}\right)}{\partial \theta_{i}} \cdot \frac{\partial \log f_{i t}\left(\psi_{0}, \lambda_{i 0}\right)}{\partial \theta_{i}^{\prime}}\right]=\left(\begin{array}{cc}
\mathcal{I}_{i, \psi \psi} & \mathcal{I}_{i, \psi \lambda} \\
\mathcal{I}_{i, \lambda \psi} & \mathcal{I}_{i, \lambda \lambda}
\end{array}\right),
$$

where the partition is conformable with $\theta_{i}=\left(\psi^{\prime}, \lambda_{i}\right)^{\prime} \in \mathbb{R}^{r+1}$. The matrices $\mathcal{I}_{i}, \mathcal{I}_{i, \psi \psi}$ and $\mathcal{I}_{i, \lambda \lambda}$ are all nonsingular from Assumption 2. We also define the (scaled individual) score 
functions as

$$
\begin{aligned}
u_{i}\left(\psi, \lambda_{i}\right) & =\frac{\partial}{\partial \psi} \ell_{i}\left(\psi, \lambda_{i}\right), \\
v_{i}\left(\psi, \lambda_{i}\right) & =\frac{\partial}{\partial \lambda_{i}} \ell_{i}\left(\psi, \lambda_{i}\right), \\
u_{i}^{e}\left(\psi, \lambda_{i}\right) & =u_{i}\left(\psi, \lambda_{i}\right)-\mathcal{I}_{i, \psi \lambda} \mathcal{I}_{i, \lambda \lambda}^{-1} v_{i}\left(\psi, \lambda_{i}\right) .
\end{aligned}
$$

Note that $u_{i}^{e}\left(\psi_{0}, \lambda_{i 0}\right)$ is the efficient score for $\psi$ at $\left(\psi_{0}, \lambda_{i 0}\right)$ and can be understood as the orthogonal projection of the score function for $\psi$ on the space spanned by the components of the nuisance score $v_{i}\left(\psi_{0}, \lambda_{i 0}\right)$ (e.g., Murphy and van der Vaart (2000) $) .7$ It can be shown that we have the following expansion (e.g., McCullagh and Tibshirani (1990), Severini (2000) and Sartori (2003)):

$$
\frac{\partial \ell_{i}^{P}\left(\psi_{0}\right)}{\partial \psi}=u_{i}^{e}\left(\psi_{0}, \lambda_{i 0}\right)+b_{i}\left(\psi_{0}\right)+O_{p}\left(\frac{1}{T^{3 / 2}}\right)
$$

with $u_{i}^{e}\left(\psi_{0}, \lambda_{i 0}\right)=O_{p}\left(T^{-1 / 2}\right)$ and $b_{i}\left(\psi_{0}\right)=O_{p}\left(T^{-1}\right)$ for all $i$. Though $\mathbb{E}_{G_{i}}\left[u_{i}^{e}\left(\psi_{0}, \lambda_{i 0}\right)\right]=0$ by construction, $\mathbb{E}_{G_{i}}\left[b_{i}\left(\psi_{0}\right)\right] \neq 0$, which leads to an asymptotic bias that appears in 10 . The modification term $M_{i}(\psi)$ in (5) can be found as a function in $\psi$, provided that $f\left(\cdot ; \theta_{i}\right)$ is thrice differentiable in $\theta_{i}$, satisfying

$$
\mathbb{E}_{G_{i}}\left[\frac{1}{T} \frac{d M_{i}\left(\psi_{0}\right)}{d \psi}-b_{i}\left(\psi_{0}\right)\right]=O\left(\frac{1}{T^{3 / 2}}\right)
$$

so that the expected score of the modified profile likelihood $\mathbb{E}_{G_{i}}\left[\partial \ell_{i}^{M}\left(\psi_{0}\right) / \partial \psi\right]$ does not have the first order asymptotic bias from $b_{i}\left(\psi_{0}\right)$.

\subsection{Bias reduction}

The standard bias corrected estimators in nonlinear (dynamic) fixed effect regressions correspond to $\widetilde{\psi}$ in $(6)$ and are given by (e.g., Hahn and Newey (2004); Arellano and Hahn (2007);

\footnotetext{
${ }^{7}$ It follows that $\mathbb{E}_{G_{i}}\left[\partial u_{i}^{e}\left(\psi_{0}, \lambda_{i 0}\right) / \partial \lambda_{i}\right]=0$ since $u_{i}^{e}\left(\psi, \lambda_{i}\right)$ and $v_{i}\left(\psi, \lambda_{i}\right)$ are orthogonal at $\left(\psi_{0}, \lambda_{i 0}\right)$ by construction (e.g., Arellano and Hahn (2007)).
} 
Hahn and Kuersteiner (2011))

$$
\widetilde{\psi}=\widehat{\psi}-\frac{1}{T}\left(\frac{1}{n} \sum_{i=1}^{n} \widehat{\mathcal{I}}_{i}^{e}\right)^{-1}\left(\frac{1}{n} \sum_{i=1}^{n} \frac{d}{d \psi} M_{i}(\widehat{\psi})\right)
$$

where $\widehat{\mathcal{I}}_{i}^{e}$ is a consistent estimator of the efficient information $\mathcal{I}_{i}^{e}=\mathcal{I}_{i, \psi \psi}-\mathcal{I}_{i, \psi \lambda} \mathcal{I}_{i, \lambda \lambda}^{-1} \mathcal{I}_{i, \lambda \psi}$. In principle, $\widehat{\mathcal{I}}_{i}^{e}$ can be derived as $-(1 / T) \sum_{t=1}^{T} \partial^{2} \log f_{i t}^{M}(\psi) / \partial \psi \partial \psi^{\prime}$ at $\psi=\widehat{\psi}$ under stationarity, where the second derivative of $\log f_{i t}^{M}(\psi)$ may need to be obtained numerically. Alternatively, it can be derived using the elements of $(1 / T) \sum_{t=1}^{T} \partial \log f_{i t}\left(\theta_{i}\right) / \partial \theta_{i} \cdot \partial \log f_{i t}\left(\theta_{i}\right) / \partial \theta_{i}^{\prime}$ at $\theta_{i}=$ $\left(\widehat{\psi}^{\prime}, \widehat{\lambda}_{i}(\widehat{\psi})\right)^{\prime}$, which estimates $\mathcal{I}_{i}$ in 11$)$. The expression of $d M_{i}(\widehat{\psi}) / d \psi$ can be derived in the same way as equation (12) in Arellano and Hahn (2007).

For later use, we can derive a simple form of $M_{i}(\psi)$ as follows under the regularity conditions and Assumptions 1 and 2. From standard asymptotic results for (Q)ML estimators, we have the first order stochastic expansion for an arbitrary fixed $\psi$ as

$$
\sqrt{T}\left(\widehat{\lambda}_{i}(\psi)-\lambda_{i}(\psi)\right)=\overline{\mathcal{H}}_{i}(\psi)^{-1} \cdot \sqrt{T} \frac{\partial \ell_{i}\left(\psi, \lambda_{i}(\psi)\right)}{\partial \lambda_{i}}+O_{p}\left(\frac{1}{T^{1 / 2}}\right)
$$

for each $i$, where $\overline{\mathcal{H}}_{i}(\psi)=\lim _{T \rightarrow \infty} \mathbb{E}_{G_{i}}\left(-\partial^{2} \ell_{i}\left(\psi, \lambda_{i}(\psi)\right) / \partial \lambda_{i}^{2}\right)$. Similarly we can expand $\ell_{i}^{P}(\psi)=\ell_{i}\left(\psi, \hat{\lambda}_{i}(\psi)\right)$ around $\lambda_{i}(\psi)$ for given $\psi$ as

$$
\begin{aligned}
\ell_{i}^{P}(\psi)-\ell_{i}\left(\psi, \lambda_{i}(\psi)\right)= & \frac{\partial \ell_{i}\left(\psi, \lambda_{i}(\psi)\right)}{\partial \lambda_{i}}\left(\widehat{\lambda}_{i}(\psi)-\lambda_{i}(\psi)\right) \\
& -\frac{1}{2} \overline{\mathcal{H}}_{i}(\psi)\left(\widehat{\lambda}_{i}(\psi)-\lambda_{i}(\psi)\right)^{2}+O_{p}\left(\frac{1}{T^{3 / 2}}\right) \\
= & \frac{1}{2 T} \overline{\mathcal{H}}_{i}(\psi)^{-1}\left(\sqrt{T} \frac{\partial \ell_{i}\left(\psi, \lambda_{i}(\psi)\right)}{\partial \lambda_{i}}\right)^{2}+O_{p}\left(\frac{1}{T^{3 / 2}}\right)
\end{aligned}
$$

from 14 , where the dominating term is $O_{p}\left(T^{-1}\right)$ because $\overline{\mathcal{H}}_{i}(\psi)=O(1)$ and $\partial \ell_{i}\left(\psi, \lambda_{i}(\psi)\right) / \partial \lambda_{i}=$ $O_{p}\left(T^{-1 / 2}\right)$. Since $\lambda_{i}\left(\psi_{0}\right)=\lambda_{i 0}$ and $\mathbb{E}_{G_{i}}\left[\partial \ell_{i}\left(\psi_{0}, \lambda_{i 0}\right) / \partial \psi\right]=0$ by construction, 12 and 13 . suggest that the modification term can be derived as a consistent estimator of the bias term:

$$
\mathbb{E}_{G_{i}}\left[\ell_{i}^{P}\left(\psi_{0}\right)-\ell_{i}\left(\psi_{0}, \lambda_{i 0}\right)\right]=\frac{1}{2 T} \overline{\mathcal{H}}_{i}\left(\psi_{0}\right)^{-1} \mathbb{E}_{G_{i}}\left[\left(\sqrt{T} \frac{\partial \ell_{i}\left(\psi_{0}, \lambda_{i 0}\right)}{\partial \lambda_{i}}\right)^{2}\right]+O\left(\frac{1}{T^{3 / 2}}\right)
$$


For example, a simple form of the modification function in $\ell_{i}^{M}(\psi)$ is obtained as

$$
\begin{aligned}
\frac{1}{T} M_{i}(\psi)= & \frac{1}{2 T}\left(-\frac{1}{T} \sum_{t=1}^{T} \frac{\partial^{2} \log f_{i t}\left(\psi, \widehat{\lambda}_{i}(\psi)\right)}{\partial \lambda_{i}^{2}}\right)^{-1} \\
& \times \sum_{j=-m}^{m} \frac{K_{j}}{T} \sum_{t=\max \{1, j+1\}}^{\min \{T, T+j\}} \frac{\partial \log f_{i t}\left(\psi, \widehat{\lambda}_{i}(\psi)\right)}{\partial \lambda_{i}} \cdot \frac{\partial \log f_{i t-j}\left(\psi, \widehat{\lambda}_{i}(\psi)\right)}{\partial \lambda_{i}},
\end{aligned}
$$

whose first derivative corrects the leading bias term $b_{i}\left(\psi_{0}\right)$ at $\psi=\psi_{0}$ in the profile score (12) with probability approaching to one. The second component in (17) corresponds to the robust variance estimator of $\sqrt{T} \partial \ell_{i}\left(\psi, \widehat{\lambda}_{i}(\psi)\right) / \partial \lambda_{i}$. For a more general treatment of the modification to the profile likelihood, see Barndorff-Nielsen (1983) for the modified profile likelihood approach or McCullagh and Tibshirani (1990) for the adjusted profile likelihood approach. Note that $M_{i}(\psi) / T$ in $(17)$ is similar to the modification functions suggested by Arellano and Hahn (2006) and Bester and Hansen (2009), which appears to be robust to arbitrary serial correlation in $\partial \log f_{i t}\left(\psi, \widehat{\lambda}_{i}(\psi)\right) / \partial \lambda_{i}$. The truncation parameter $m \geq 0$ is chosen so that $m / T^{1 / 2} \rightarrow 0$ as $T \rightarrow \infty$, and the lag kernel function $K_{j}$ generally guarantees positive definiteness of the variance estimate (e.g., by use of the Bartlett kernel: $K_{j}=$ $1-(j /(m+1)))$.

\section{Profile Likelihood and KLIC}

\subsection{Model selection}

Panel studies conventionally focus on reducing the first order bias (10) arising from the presence of incidental parameters under a presumption that the models are correctly specified. As discussed in Lee (2006, 2012), however, if the model is not correctly specified, effort to reduce bias due to incidental parameters may be counterproductive and even exacerbate bias. Achieving correct model specification is therefore an important component in successful bias reduction, particularly for dynamic and nonlinear panel models. Examples include the choice of lag order in panel $A R M A$ models or the functional structure in nonlinear panels. Importantly, correct model specification should precede the use of any bias corrections. We 
focus here on model specification - in particular, we are interested in selecting a model $f\left(z ; \psi, \lambda_{i}\right)$ that is closest to the true model $g_{i}(z)$ on average over $i$.

Intuitively, when there are no nuisance parameters $\lambda_{i}$ so that the dimension of the parameter vector $\theta=\psi$ is small and finite, we can conduct standard model selection by comparing estimates of the averaged KLIC given by

$$
\begin{aligned}
\min _{\theta} \bar{D}(g \| f(\theta)) & =\min _{\theta} \lim _{n \rightarrow \infty} \frac{1}{n} \sum_{i=1}^{n} D\left(g_{i}(\cdot) \| f(\cdot ; \theta)\right) \\
& =\min _{\theta} \lim _{n \rightarrow \infty} \frac{1}{n} \sum_{i=1}^{n} \int\left\{\log g_{i}(z)-\log f(z ; \theta)\right\} d G_{i}(z)
\end{aligned}
$$

Note that averaged KLIC $\bar{D}(g \| f(\theta))$ is defined so that it accommodates possibly heterogeneous panel models. Individual $\operatorname{KLIC~} D\left(g_{i}(\cdot) \| f(\cdot ; \theta)\right)$ is nonnegative for all $i$ and the selected model $f(\cdot ; \theta)$ in this case yields the smallest sum of KLIC's over $g_{i}(\cdot)$. In practice, however, the average KLIC minimizer $\theta_{0}=\arg \min _{\theta} \lim _{n \rightarrow \infty} \bar{D}(g \| f(\theta))$ in $(18)$ is not available, and it is natural to consider the QMLE $\widehat{\theta}$, which is a consistent estimator of $\theta_{0}$. Since the first term $\log g_{i}(z)$ in 18$)$ does not depend on the model, we thus select the model $f(\cdot ; \theta)$ minimizing the relative distance

$$
\Phi(\widehat{\theta})=-\frac{1}{n} \sum_{i=1}^{n} \int \log f(z ; \widehat{\theta}) d G_{i}(z)
$$

which can be estimated by

$$
\widehat{\Phi}(\widehat{\theta})=-\frac{1}{n} \sum_{i=1}^{n} \int \log f(z ; \widehat{\theta}) d \widehat{G}_{i}(z)=-\frac{1}{n T} \sum_{i=1}^{n} \sum_{t=1}^{T} \log f\left(z_{i, t} ; \widehat{\theta}\right)
$$

under stationarity over $t$, where $\widehat{G}_{i}$ is the empirical distribution. As noted in Akaike (1973), however, $-\widehat{\Phi}(\widehat{\theta})$ overestimates $-\Phi(\widehat{\theta})$ since $\widehat{G}_{i}$ corresponds more closely to $\widehat{\theta}$ than does the true $G_{i}$. Therefore, it is suggested to minimize the bias-corrected version of $\widehat{\Phi}(\widehat{\theta})$ given by

$$
\widetilde{\Phi}(\widehat{\theta})=\widehat{\Phi}(\widehat{\theta})-\widehat{B}(\widehat{\theta})
$$


as an information criterion for model selection, where $\widehat{B}(\widehat{\theta})$ is the sample analogue of $B(\widehat{\theta})=$ $\mathbb{E}[\widehat{\Phi}(\widehat{\theta})-\Phi(\widehat{\theta})]$ and $\mathbb{E}[\cdot]$ is the expectation with respect to the joint distribution $G=\left(G_{1}, \cdots, G_{n}\right)^{\prime}$. See, for example, Akaike (1973, 1974) for further details. Note that Akaike (1973) shows that $B(\widehat{\theta})$ is asymptotically the ratio of $\operatorname{dim}(\theta)$ to the sample size when $\widehat{\theta}$ is the QMLE and $g$ is nested in $f$.

Now consider the case with incidental parameters $\lambda=\left(\lambda_{1}, \cdots, \lambda_{n}\right)^{\prime} \in \mathbb{R}^{n}$ and we let $\theta=\left(\psi^{\prime}, \lambda^{\prime}\right)^{\prime}$. Similar to the discussion of the previous section, when the dimension of the parameter vector $\theta$ is substantial relative to the sample size, the incidental parameter problem prevails and it is not straightforward to use a standard criterion based on 19 . One possible solution is to reduce the dimension of the parameters by concentrating out the nuisance parameters. Particularly when it is assumed that the (finite-dimensional) parameter of central interest $\psi$ governs the key structure of the model that is unchanging over $i$, it is natural to focus on the sub-parameter $\psi$ by concentrating out the nuisance parameters $\lambda_{i}$ in conducting model selection. The candidate models are indexed by $\psi$ alone, while the parameter space of $\lambda_{i}$ remains the same across them, and thus the choice of a particular model does not depend on the realization of $\lambda_{i}$ 's in this case. This idea is similar to the profile likelihood approach when interest lies in a subset of parameters. Some examples are as follows.

Example 1 (Variable or model selection in panel models) Consider a nonlinear fixed-effect model given by $y_{i, t}=\xi\left(x_{i, t}, u_{i, t} ; \mu_{i}, \beta, \sigma_{i}^{2}\right)$ where $\xi(\cdot ; \cdot)$ is some known specified function, $u_{i, t}$ is independent over $i$ and $t$ with $u_{i, t} \mid\left(x_{i, 1}, \cdots, x_{i, T}, \mu_{i}\right) \sim\left(0, \sigma_{i}^{2}\right)$, and $\beta$ is an $r$-dimensional parameter vector. The goal in this case is either to select a set of regressors or to choose a parametric function $\xi(\cdot ; \cdot)$ yielding the best fit in the presence of incidental parameters $\left(\mu_{i}, \sigma_{i}^{2}\right)$. For $\xi(\cdot ; \cdot)$, a common choice would be between Logit and Probit models. Variable selection in a linear transformation model given by $\varphi_{i}\left(y_{i, t}\right)=x_{i, t}^{\prime} \beta+u_{i, t}$ with some strictly increasing incidental function $\varphi_{i}(\cdot)$ is another example.

Example 2 (Lag order selection in dynamic panel regressions) Consider a panel $A R(p)$ model 
with fixed effects given by $y_{i, t}=\mu_{i}+\sum_{j=1}^{p} \alpha_{p j} y_{i, t-j}+\varepsilon_{i, t}$, where $\varepsilon_{i, t}$ is independent across $i$ and serially uncorrelated. The goal here is to choose the correct lag order $p$, allowing for the presence of incidental parameters $\mu_{i}$. When $p=\infty$, the problem becomes one of finding a best approximation in the finite order $A R(p)$ class.

Example 3 (Number of support choice of random effects or random coefficient) Consider a random-effect/coefficient model given by $y_{i, t}=x_{i, t}^{\prime} \beta_{i}+\varepsilon_{i, t}$, where $\varepsilon_{i, t}$ is independent over $i$ and $t$ with $\varepsilon_{i, t} \mid\left(x_{i, 1}, \cdots, x_{i, T}, \beta_{i}\right) \sim \mathcal{N}\left(0, \sigma_{i}^{2}\right)$, and $\beta_{i}$ is an i.i.d. unobserved random variable independent of $x_{i, t}$ and $\varepsilon_{i, t}$ with a common distribution over the finite support $\left\{q_{1}, \cdots, q_{k}\right\}$. The main interest in this example is to determine the finite support number $k$ in the presence of incidental parameters $\sigma_{i}^{2}$. In the context of mixed proportional hazard models (or Cox partial likelihoods with unobserved heterogeneity), the problem is to choose the finite support number of nonparametric frailty in the Heckman-Singer model (Heckman and Singer (1984)), if the Cox partial likelihood is viewed as a profile likelihood.

\subsection{Profile likelihood information criterion}

For model selection using an information criterion in the presence of incidental parameters we consider the profile Kullback-Leibler divergence, in which the incidental parameters $\lambda_{i}$ are concentrated out of the standard KLIC as follows.

Definition (Profile KLIC) The profile Kullback-Leibler divergence (or the profile KLIC) of $g_{i}(\cdot)$ relative to $f\left(\cdot ; \psi, \lambda_{i}\right)$ is defined as

$$
D_{P}\left(g_{i}(\cdot) \| f\left(\cdot ; \psi, \lambda_{i}\right) ; \psi\right)=\min _{\lambda_{i} \in \Lambda} D\left(g_{i}(\cdot) \| f\left(\cdot ; \psi, \lambda_{i}\right)\right)
$$

Note that $D_{P}\left(g_{i}(\cdot) \| f\left(\cdot ; \psi, \lambda_{i}\right) ; \psi\right)$ depends on $\psi$ only, not on $\lambda_{i}$. Since the profile KLIC is defined as the minimum of the standard $\operatorname{KLIC~} D\left(g_{i}(\cdot) \| f\left(\cdot ; \psi, \lambda_{i}\right)\right)$ in $\lambda_{i}$, it apparently satisfies the same conditions as standard KLIC. For example, $D_{P}\left(g_{i}(\cdot) \| f\left(\cdot ; \psi, \lambda_{i}\right) ; \psi\right)$ is nonnegative and equals zero when $g_{i}(\cdot)$ belongs to the parametric family of $f\left(\cdot ; \psi, \lambda_{i}\right)$ (i.e., 
$g_{i}(\cdot)=f\left(\cdot ; \psi_{*}, \lambda_{i *}\right)$ for some $\left.\left(\psi_{*}^{\prime}, \lambda_{i *}\right)^{\prime} \in \Psi \times \Lambda\right)$.

Similar to the standard case (18), we select the model that has the smallest value of the estimate of

$$
\begin{aligned}
\min _{\psi \in \Psi} \bar{D}_{P}(g \| f(\psi, \lambda) ; \psi) & =\min _{\psi \in \Psi} \lim _{n \rightarrow \infty} \frac{1}{n} \sum_{i=1}^{n} D_{P}\left(g_{i}(\cdot) \| f\left(\cdot ; \psi, \lambda_{i}\right) ; \psi\right) \\
& =\min _{\psi \in \Psi} \lim _{n \rightarrow \infty} \frac{1}{n} \sum_{i=1}^{n} D\left(g_{i}(\cdot) \| f\left(\cdot ; \psi, \lambda_{i}(\psi)\right)\right)
\end{aligned}
$$

where the last expression is from the definition of $\lambda_{i}(\psi)$ given in (8). From the definition in (20), note that the model with the smallest (21) can be understood as the model with the smallest averaged KLIC over $\psi$ and $\lambda=\left(\lambda_{1}, \cdots, \lambda_{n}\right)^{\prime}$.

In practice, we cannot directly use $(21)$ for model selection since the average KLIC minimizers $\psi_{0}=\arg \min _{\psi \in \Psi} \lim _{n \rightarrow \infty}(1 / n) \sum_{i=1}^{n} D\left(g_{i}(\cdot) \| f\left(\cdot ; \psi, \lambda_{i}(\psi)\right)\right)$ in $(9)$ and $\lambda_{i 0}=\lambda_{i}\left(\psi_{0}\right)$ in (8) are not available. For $\psi$, it is natural to use the quasi maximum modified profile likelihood estimator (i.e., the bias-corrected estimator) $\widetilde{\psi}$ in $(6)$ as discussed in the previous section; whereas for $\lambda_{i}(\psi)$, a natural candidate is $\hat{\lambda}_{i}(\psi)$ in $(3)$. Therefore, instead of $\min _{\psi \in \Psi} \bar{D}_{P}(g \| f(\psi, \lambda) ; \psi)$ in $(21)$, we consider the averaged KLIC based on the profile likelihoods given by

$$
\bar{D}\left(g \| f^{P}(\widetilde{\psi})\right)=\bar{D}(g \| f(\widetilde{\psi}, \widehat{\lambda}(\widetilde{\psi})))=\frac{1}{n} \sum_{i=1}^{n} D\left(g_{i}(\cdot) \| f\left(\cdot ; \widetilde{\psi}, \widehat{\lambda}_{i}(\widetilde{\psi})\right)\right)
$$

Since $\widehat{\lambda}_{i}(\psi)$ is a biased estimator of $\lambda_{i}(\psi)$ when $T$ is small, however, the KLIC based on the profile likelihoods $D\left(g_{i}(\cdot) \| f\left(\cdot ; \psi, \widehat{\lambda}_{i}(\psi)\right)\right)$ in 22 is in general different from the profile KLIC $D_{P}\left(g_{i}(\cdot) \| f\left(\cdot ; \psi, \lambda_{i}\right) ; \psi\right)=D\left(g_{i}(\cdot) \| f\left(\cdot ; \psi, \lambda_{i}(\psi)\right)\right)$ in $(20)$. The following lemma states the relation between these two KLIC's.

Lemma 1 For a given $\psi \in \Psi$, we have

$$
D_{P}\left(g_{i}(\cdot) \| f\left(\cdot ; \psi, \lambda_{i}\right) ; \psi\right)=D\left(g_{i}(\cdot) \| f\left(\cdot ; \psi, \widehat{\lambda}_{i}(\psi)\right)\right)+\delta_{i}(\psi)
$$


for each $i$, where the bias term is defined as $\delta_{i}(\psi)=\mathbb{E}_{G_{i}}\left[\ell_{i}^{P}(\psi)-\ell_{i}\left(\psi, \lambda_{i}(\psi)\right)\right]$. Furthermore, if Assumptions 1 and 2 hold, $\delta_{i}(\psi)$ satisfies

$$
\mathbb{E}_{G_{i}}\left[\delta_{i}\left(\psi_{0}\right)-\left(\frac{M_{i}\left(\psi_{0}\right)}{T}\right)\right]=O\left(\frac{1}{T^{3 / 2}}\right)
$$

under the regularity conditions, where $M_{i}(\psi)$ is the modification term used for the modified profile likelihood function (5).

Even when $g_{i}$ is nested in $f$, 23) shows that $D\left(g_{i}(\cdot) \| f\left(\cdot ; \psi_{0}, \widehat{\lambda}_{i}\left(\psi_{0}\right)\right)\right)$ is not necessarily zero unless $f\left(z ; \psi_{0}, \lambda_{i}\left(\psi_{0}\right)\right)=f\left(z ; \psi_{0}, \widehat{\lambda}_{i}\left(\psi_{0}\right)\right)$, which is unlikely with small $T$. Since the ideal (infeasible) model selection is based on $D_{P}\left(g_{i}(\cdot) \| f\left(\cdot ; \psi, \lambda_{i}\right) ; \psi\right)$, it follows that model selection using $D\left(g_{i}(\cdot) \| f\left(\cdot ; \psi, \widehat{\lambda}_{i}(\psi)\right)\right)$ suffers distortion and should be modified by correcting the bias using a suitable estimator of $\delta_{i}(\psi)$. The result in 24 shows that the bias term in 23 is indeed closely related to the modification term $M_{i}(\psi)$.

Similar to 19 , therefore, by letting

$$
\Phi_{P}(\widetilde{\psi})=-\frac{1}{n} \sum_{i=1}^{n} \int \log f\left(z ; \widetilde{\psi}, \widehat{\lambda}_{i}(\widetilde{\psi})\right) d G_{i}(z)
$$

we define an information criterion using a bias-corrected estimator of $\Phi_{P}(\widetilde{\psi})$ given by

$$
\widetilde{\Phi}_{P}(\widetilde{\psi})=-\frac{1}{n T} \sum_{i=1}^{n} \sum_{t=1}^{T} \log f_{i t}^{P}(\widetilde{\psi})-\left\{\widehat{B}_{P}(\widetilde{\psi})-\frac{1}{n T} \sum_{i=1}^{n} M_{i}(\widetilde{\psi})\right\},
$$

where $f_{i t}^{P}(\widetilde{\psi})=f\left(z_{i, t} ; \widetilde{\psi}, \widehat{\lambda}_{i}(\widetilde{\psi})\right)$ and $\widehat{B}_{P}(\widetilde{\psi})$ is the sample analogue of

$$
B_{P}(\widetilde{\psi})=\mathbb{E}\left[-\frac{1}{n} \sum_{i=1}^{n} \int \log f\left(z ; \widetilde{\psi}, \widehat{\lambda}_{i}(\widetilde{\psi})\right) d\left(\widehat{G}_{i}(z)-G_{i}(z)\right)\right]
$$

$\widetilde{\Phi}_{P}(\widetilde{\psi})$ in 25 includes two bias correction terms: $\widehat{B}_{P}(\widetilde{\psi})$ is introduced from the same reason as the standard model selection cases; the additional correction term $-(n T)^{-1} \sum_{i=1}^{n} M_{i}(\widetilde{\psi})$ is introduced from Lemma 1 because the feasible information criterion is defined using $D\left(g_{i}(\cdot) \| f\left(\cdot ; \psi, \widehat{\lambda}_{i}(\psi)\right)\right)$ instead of $D_{P}\left(g_{i}(\cdot) \| f\left(\cdot ; \psi, \lambda_{i}\right) ; \psi\right)$. The following theorem derives 
an approximate expression for $B_{P}(\widetilde{\psi})$ based on which the information criterion is to be developed. We denote $z_{i}=\left(z_{i, 1}, \cdots, z_{i, T}\right)^{\prime}$.

Theorem 2 Let Assumptions 1 and 2 hold. If $n, T \rightarrow \infty$ satisfying $n / T \rightarrow \kappa \in(0, \infty)$, under regularity conditions (given for example in Hahn and Kuersteiner (2011)), we have

$$
B_{P}(\widetilde{\psi})=-\frac{1}{n T} \operatorname{tr}\left\{J(G)^{-1} I(G)\right\}+o\left(\frac{1}{n T}\right)
$$

where $\operatorname{tr}\{\cdot\}$ is the trace operator and

$$
\begin{aligned}
J(G) & =\frac{1}{n} \sum_{i=1}^{n} \mathbb{E}_{G_{i}}\left[-\left.\frac{1}{T} \sum_{t=1}^{T} \frac{\partial^{2} \log f\left(z_{i, t} ; \psi, \lambda_{i}(\psi)\right)}{\partial \psi \partial \psi^{\prime}}\right|_{\psi=H(G)}\right], \\
I(G) & =\frac{1}{n} \sum_{i=1}^{n} \mathbb{E}_{G_{i}}\left[\left.\left.\frac{1}{T} \sum_{t=1}^{T} \sum_{s=1}^{T} \frac{\partial \log f\left(z_{i, t} ; \psi, \lambda_{i}(\psi)\right)}{\partial \psi}\right|_{\psi=H(G)} \frac{\partial \log f\left(z_{i, s} ; \psi, \hat{\lambda}_{i}(\psi)\right)}{\partial \psi^{\prime}}\right|_{\psi=H(G)}\right] .
\end{aligned}
$$

Note that $I(G)$ is defined by the product of the score functions of $f\left(z ; \psi, \lambda_{i}(\psi)\right)$ and $f^{P}(z ; \psi)=$ $f\left(z ; \psi, \widehat{\lambda}_{i}(\psi)\right)$. For some truncation parameter $m \geq 0$ such that $m / T^{1 / 2} \rightarrow 0$ as $T \rightarrow \infty$ and a properly chosen lag kernel function $K_{j}$ similarly as $M_{i}(\psi)$, we can obtain a consistent estimator for $B_{P}(\widetilde{\psi})$ as

$$
\widehat{B}_{P}(\widetilde{\psi})=-\frac{1}{n T} \operatorname{tr}\left\{J(\widehat{G})^{-1} I(\widehat{G})\right\}
$$

where

$$
\begin{aligned}
J(\widehat{G}) & =-\frac{1}{n T} \sum_{i=1}^{n} \sum_{t=1}^{T} \frac{\partial^{2} \log f_{i t}^{M}(\widetilde{\psi})}{\partial \psi \partial \psi^{\prime}} \text { and } \\
I(\widehat{G}) & =\frac{1}{n T} \sum_{i=1}^{n} \sum_{j=-m}^{m} K_{j} \sum_{t=\max \{1, j+1\}}^{\min \{T, T+j\}} \frac{\partial \log f_{i t}^{M}(\widetilde{\psi})}{\partial \psi} \frac{\partial \log f_{i t-j}^{P}(\widetilde{\psi})}{\partial \psi^{\prime}} .
\end{aligned}
$$

From equations (25) and (26), therefore, a general form of information criterion for model selection based on the bias-corrected profile likelihood (i.e., a profile likelihood information 
criterion; PLIC) may be defined as

$$
\begin{aligned}
P L I C(f)= & -\frac{2}{n T} \sum_{i=1}^{n} \sum_{t=1}^{T} \log f_{i t}^{P}(\widetilde{\psi})-2\left\{\widehat{B}_{P}(\widetilde{\psi})-\frac{1}{n T} \sum_{i=1}^{n} M_{i}(\widetilde{\psi})\right\} \\
= & -\frac{2}{n T} \sum_{i=1}^{n} \sum_{t=1}^{T} \log f\left(z_{i, t} ; \widetilde{\psi}, \widehat{\lambda}_{i}(\widetilde{\psi})\right) \\
& +\frac{2}{n T} \operatorname{tr}\left\{J(\widehat{G})^{-1} I(\widehat{G})\right\}+\frac{2}{n T} \sum_{i=1}^{n} M_{i}(\widetilde{\psi})
\end{aligned}
$$

where $M_{i}(\widetilde{\psi})$ is given by $(17)$ in general. This new information criterion includes two penalty terms. The first penalty term corresponds to the standard finite sample adjustment as in AIC, whereas the second penalty term reflects bias correction from using the profile likelihood in the model selection problem. With further conditions, we can derive a simpler form for $P L I C(f)$ as shown in the following corollary.

Corollary 3 Suppose that $g_{i}(\cdot)$ is included in the family of $f\left(\cdot ; \psi, \lambda_{i}\right)$. Under the same conditions as Theorem 2, we have

$$
P L I C(f)=-\frac{2}{n T} \sum_{i=1}^{n} \sum_{t=1}^{T} \log f\left(z_{i, t} ; \widetilde{\psi}, \widehat{\lambda}_{i}(\widetilde{\psi})\right)+\frac{2 r}{n T}+\frac{2}{n T} \sum_{i=1}^{n} M_{i}(\widetilde{\psi})
$$

where $r=\operatorname{dim}(\psi)$.

Note that the goodness-of-fit is based on the maximized profile likelihood, which corresponds to the standard maximized likelihood though it is evaluated at $\widetilde{\psi}$ instead of at the MLE. The additional penalty term $(2 / n T) \sum_{i=1}^{n} M_{i}(\widetilde{\psi})$ is novel and is nonzero in the presence of incidental parameters. Since this additional penalty term is positive by construction, the new information criterion (27) or (28) has heavier penalty than the standard Akaike information criterion (AIC). Since $(2 / n T) \sum_{i=1}^{n} M_{i}(\widetilde{\psi})=O_{p}\left(T^{-1}\right)$, the second penalty term can dominate the first one by a large margin when $n$ is quite large. Recall that in the standard AIC, this additional penalty term does not appear and the penalty term of the information criterion is simply given by $2 r / n T$ via a standardized parameter count. 


\section{Integrated Likelihood and Bayesian Approach}

Instead of a KLIC-based model selection criteria using the (modified) profile likelihood, we next consider a Bayesian approach using the integrated likelihood (e.g., Berger et al. (1999)). The result in this section shows that the difference between the integrated likelihood based approach and the profile likelihood based approach lies in their penalty terms, where the penalty terms are of the same form as standard AIC and BIC cases.

We first assume a conditional prior of $\lambda_{i}$ as $\pi_{i}\left(\lambda_{i} \mid \psi\right)$ for each $i$, which satisfies the following conditions, as in Arellano and Bonhomme (2009):

Assumption 3 (i) The support of $\pi_{i}\left(\lambda_{i} \mid \psi\right)$ contains an open neighborhood of $\left(\psi_{0}, \lambda_{i 0}\right)$.

(ii) When $T \rightarrow \infty, \log \pi_{i}\left(\lambda_{i} \mid \psi\right)=O(1)$ uniformly over $i$ for all $\lambda_{i}$ and $\psi$.

Using $\pi_{i}\left(\lambda_{i} \mid \psi\right)$, the individual integrated log-likelihood $\ell_{i}^{I}(\psi)$ is defined as

$$
\ell_{i}^{I}(\psi)=\frac{1}{T} \log \left\{\int f_{i}\left(\psi, \lambda_{i}\right) \pi_{i}\left(\lambda_{i} \mid \psi\right) d \lambda_{i}\right\}
$$

for each $i$, where $f_{i}\left(\psi, \lambda_{i}\right)=\prod_{t=1}^{T} f_{i t}\left(\psi, \lambda_{i}\right)=\exp \left(T \ell_{i}\left(\psi, \lambda_{i}\right)\right)$ is the joint density of $z_{i}=$ $\left(z_{i, 1}, \cdots, z_{i, T}\right)^{\prime}$. Let $\phi^{k}$ be the discrete prior over different $K$ models $\mathcal{M}^{1}, \mathcal{M}^{2}, \cdots, \mathcal{M}^{K}$ and $\eta\left(\psi^{k} \mid \mathcal{M}^{k}\right)$ be the prior on $\psi^{k} \in \mathbb{R}^{r_{k}}$ given the model $\mathcal{M}^{k}$. Further, let $g(z)=\prod_{i=1}^{n} g_{i}\left(z_{i}\right)$ be the joint density of $\left(z_{1}, \cdots, z_{n}\right)$ and

$$
L^{I}\left(\psi^{k} \mid z\right)=\exp \left(T \sum_{i=1}^{n} \ell_{i}^{I}\left(\psi^{k}\right)\right)
$$

be the integrated (joint) likelihood function. Then, Bayes theorem yields the posterior probability of the model $\mathcal{M}^{k}$ as

$$
\mathcal{P}\left(\mathcal{M}^{k} \mid z\right)=\frac{1}{g(z)} \phi^{k} \int L^{I}\left(\psi^{k} \mid z\right) \eta\left(\psi^{k} \mid \mathcal{M}^{k}\right) d \psi^{k}
$$

and the Bayesian information criterion can be obtained based on $-2 \log \mathcal{P}\left(\mathcal{M}^{k} \mid z\right)$. By choosing the candidate model corresponding to the minimum value of the Bayesian information 
criterion, the goal is to select the candidate model corresponding to the highest Bayesian posterior probability. This approach is approximately equivalent to model selection based on Bayes factors (e.g., Kass and Raftery (1995)).

Note from Lemma 1 of Arellano and Bonhomme (2009), we can link the integrated and the (modified) profile likelihood as follows using a Laplace approximation:

$\ell_{i}^{I}\left(\psi^{k}\right)-\ell_{i}^{P}\left(\psi^{k}\right)=\frac{1}{2 T} \log \left(\frac{2 \pi}{T}\right)-\frac{1}{2 T} \log \left(-\frac{\partial^{2} \ell_{i}\left(\psi^{k}, \widehat{\lambda}_{i}\left(\psi^{k}\right)\right)}{\partial \lambda_{i}^{2}}\right)+\frac{1}{T} \log \pi_{i}\left(\widehat{\lambda}_{i}\left(\psi^{k}\right) \mid \psi^{k}\right)+O_{p}\left(\frac{1}{T^{2}}\right)$

or

$$
\begin{aligned}
\ell_{i}^{I}\left(\psi^{k}\right)-\ell_{i}^{M}\left(\psi^{k}\right)= & \frac{1}{2 T} \log \left(\frac{2 \pi}{T}\right)-\frac{1}{2 T} \log \left(-\frac{\partial^{2} \ell_{i}\left(\psi^{k}, \widehat{\lambda}_{i}\left(\psi^{k}\right)\right)}{\partial \lambda_{i}^{2}}\right)+\frac{1}{T} \log \pi_{i}\left(\widehat{\lambda}_{i}\left(\psi^{k}\right) \mid \psi^{k}\right) \\
& +\frac{1}{T} M_{i}\left(\psi^{k}\right)+O_{p}\left(\frac{1}{T^{2}}\right)
\end{aligned}
$$

for each $i$. These expansions imply that if we choose the conditional prior $\pi_{i}\left(\lambda_{i} \mid \psi^{k}\right)$ such that it cancels out the leading terms in 30 at $\lambda_{i}=\widehat{\lambda}_{i}\left(\psi^{k}\right)$, then we have an improved approximation. More precisely, from (15) and (17), we obtain

$$
\begin{aligned}
\pi_{i}\left(\lambda_{i} \mid \psi^{k}\right)= & C_{\pi}\left(\mathbb{E}_{\widehat{G}_{i}}\left[-\frac{\partial^{2} \ell_{i}\left(\psi^{k}, \lambda_{i}\right)}{\partial \lambda_{i}^{2}}\right]\right)^{1 / 2} \\
& \times \exp \left\{-\frac{T}{2}\left(\mathbb{E}_{\widehat{G}_{i}}\left[-\frac{\partial^{2} \ell_{i}\left(\psi^{k}, \lambda_{i}\right)}{\partial \lambda_{i}^{2}}\right]\right)^{-1}\left(\mathbb{E}_{\widehat{G}_{i}}\left[\frac{\partial \ell_{i}\left(\psi^{k}, \lambda_{i}\right)}{\partial \lambda_{i}}\right]\right)^{2}\right\}
\end{aligned}
$$

for some positive constant $C_{\pi}$, where $\mathbb{E}_{\widehat{G}_{i}}[\cdot]$ denotes the empirical expectation for each $i$. Note that the explicit form of the conditional prior in (31) corresponds to the robust (or bias-reducing) prior in equation (14) of Arellano and Bonhomme (2009) in the case of a pseudo-likelihood. Arellano and Bonhomme (2009)'s robust prior is developed to obtain firstorder unbiased estimators in nonlinear panel models. This idea extends to our context since we find the conditional prior such that it better approximates the modified profile likelihood by the integrated likelihood, where the modified profile likelihood $\ell_{i}^{M}$ is closer to the genuine likelihood (compared with the profile likelihood $\ell_{i}^{P}$ ) and the maximum modified profile 
likelihood estimator is first-order unbiased by construction (e.g., Section 2.3). Therefore, the discussion in Arellano and Bonhomme (2009) also applies to the conditional prior $\pi_{i}\left(\lambda_{i} \mid \psi^{k}\right)$ in (31): unlike the Jeffreys' prior, it generally depends on the data unless an orthogonal reparametrization (e.g., Lancaster (2002)) or some equivalent condition is available.

By choosing the conditional prior as (31), we obtain the approximate posterior probability of the model $\mathcal{M}^{k}$ in 29$)$ as follows. We denote by $\widetilde{\psi}^{k}$ the quasi maximum modified profile likelihood estimator of $\psi^{k}$ in the model $\mathcal{M}^{k}$.

Theorem 4 Let Assumptions 1 to 3 hold and $n / T \rightarrow \kappa \in(0, \infty)$ as $n, T \rightarrow \infty$. If we suppose conditional priors of $\lambda_{i}$ as in (31) and uninformative flat priors for $\psi^{k}$ (i.e., $\eta\left(\psi^{k} \mid \mathcal{M}^{k}\right)=1$ for all $k=1, \cdots, K)$ over the neighborhood of $\widetilde{\psi}^{k}$ where $L^{I}\left(\psi^{k} \mid z\right)$ is dominant, we have the approximation

$$
\log \mathcal{P}\left(\mathcal{M}^{k} \mid z\right)=\sum_{i=1}^{n} \sum_{t=1}^{T} \log f_{i t}^{M}\left(\widetilde{\psi}^{k}\right)-\frac{r_{k}}{2} \log n T+c(z, k)+o_{p}(1)
$$

for some $c(z, k)=O_{p}(1)$, where $\log f_{i t}^{M}\left(\widetilde{\psi}^{k}\right)=\log f\left(z_{i, t} ; \widetilde{\psi}^{k}, \widehat{\lambda}_{i}\left(\widetilde{\psi}^{k}\right)\right)-M_{i}\left(\widetilde{\psi}^{k}\right) / T$ and $r_{k}=$ $\operatorname{dim}\left(\psi^{k}\right)$.

We can define the integrated likelihood information criterion $(I L I C)$ from $-(2 / n T) \log \mathcal{P}\left(\mathcal{M}^{k} \mid z\right)$ retaining only the relevant terms as follows:

$$
\operatorname{ILIC}\left(\mathcal{M}^{k}\right)=-\frac{2}{n T} \sum_{i=1}^{n} \sum_{t=1}^{T} \log f\left(z_{i, t} ; \widetilde{\psi}^{k}, \widehat{\lambda}_{i}\left(\widetilde{\psi}^{k}\right)\right)+\frac{r_{k} \log n T}{n T}+\frac{2}{n T} \sum_{i=1}^{n} M_{i}\left(\widetilde{\psi}^{k}\right) .
$$

Compared with PLIC in (28), the only difference in $(33)$ is the second term (or the first penalty term), which corresponds to the standard penalty term in BIC. This result implies that we also need to modify BIC in the presence of incidental parameters, where the correction term (i.e., the additional penalty term) is the same as the KLIC-based (AIC-type) information criteria $P L I C$ obtained in the previous section.

Therefore, in general, we can construct the following information criteria for nested mod- 
els, which can be used in the presence of incidental parameters 8

$$
\operatorname{LIC}\left(\mathcal{M}^{k}\right)=-\frac{2}{n T} \sum_{i=1}^{n} \sum_{t=1}^{T} \log f\left(z_{i, t} ; \widetilde{\psi}^{k}, \widehat{\lambda}_{i}\left(\widetilde{\psi}^{k}\right)\right)+r_{k} \frac{h(n T)}{n T}+\frac{2}{n T} \sum_{i=1}^{n} M_{i}\left(\widetilde{\psi}^{k}\right)
$$

for a candidate parametric model $\mathcal{M}^{k}$ whose parameter vector is given by $\left(\psi^{k}, \lambda_{1}, \cdots, \lambda_{n}\right)^{\prime}$ with $\operatorname{dim}\left(\psi^{k}\right)=r_{k} . h(n T)$ is some nondecreasing positive function of the sample size $n T$. The choice of $h(n T)$ is 2 for AIC-type criteria and $\log n T$ for BIC-type criteria. We conjecture that $h(n T)=2 \log \log n T$ for HQ-type criteria, although this formulation is not derived here. Note that the penalty term in $L I C$ is no longer deterministic. It is data-dependent. So this form of model selection is adaptive.

Remark (Model complexity) The trade-off between goodness-of-fit and model complexity (or penalty) is the key concept in standard model selection. The first term of the new model selection criteria (34) measures goodness-of-fit, whereas the remaining terms represent model complexity that reflects the level of difficulty of estimation (e.g., Rissanen (1986) and Hodges and Sargent (2001)). Unlike standard model selection criteria, however, the model complexity of $\operatorname{LIC}\left(\mathcal{M}^{k}\right)$ differs from the entire number of unknown parameters (i.e., $r_{k}+n$ in this case). The effect from the incidental parameters $\lambda_{i}$ can be smaller than $n$, where the degree is determined by the size of $\sum_{i=1}^{n} M_{i}\left(\widetilde{\psi}^{k}\right)$. More precisely, we can see that the model complexity measure in $\operatorname{LIC}\left(\mathcal{M}^{k}\right)$ combines the following two complexity concepts: the parametric complexity measured by the number of parameters in the focused sub-model, $r_{k}$; and the geometric complexity measured by $\sum_{i=1}^{n} M_{i}\left(\widetilde{\psi}^{k}\right)$. Since $M_{i}(\cdot)$ is a function of the Fisher information estimator and the Hessian of the profile likelihood as given in $(17), M_{i}(\cdot)$ can describe the structural complexity of the model, especially how the incidental parameters are related with the parameters $\psi^{k}$ in the focused sub-model. For a general overview of model complexity in model selection, see Bozdogan (2000), for example.

\footnotetext{
${ }^{8}$ Note that $L I C\left(\mathcal{M}^{k}\right)$ can be rewritten as $-(2 / n T) \sum_{i=1}^{n} \sum_{t=1}^{T} \log f_{i t}^{M}\left(z_{i, t} ; \widetilde{\psi}^{k}\right)+r_{k} \cdot h(n T) / n T$, where $\log f_{i t}^{M}(\cdot ; \psi)=\log f_{i t}\left(\cdot ; \psi, \widehat{\lambda}_{i}(\psi)\right)-M_{i}(\psi) / T$. This expression shows that, if the standard form of the information criteria is considered, the goodness-of-fit needs to be measured using the modified profile likelihood, which is closer to the genuine likelihood than the profile likelihood.
} 


\section{$5 \quad$ Lag Order Selection in Dynamic Panels}

\subsection{Lag order selection criteria}

As an illustration, we consider model selection criteria in the context of dynamic panel regression. In particular, we consider a panel process $\left\{y_{i, t}\right\}$ generated from the homogeneous $p_{0}$ 'th order univariate autoregressive $\left(A R\left(p_{0}\right)\right)$ model given by

$$
y_{i, t}=\mu_{i}+\sum_{j=1}^{p_{0}} \alpha_{p_{0} j} y_{i, t-j}+\varepsilon_{i, t} \text { for } i=1,2, \cdots, n \text { and } t=1,2, \cdots, T \text {. }
$$

When $p_{0}$ is finite, the goal is to pick the correct lag order $9^{9}$ The errors $\varepsilon_{i, t}$ are serially uncorrelated and the unobserved individual effects $\mu_{i}$ are assumed fixed. Let the initial values $\left(y_{i, 0}, y_{i,-1}, \cdots, y_{i,-p_{0}+1}\right)$ be observed for all $i$, which are drawn from the same distribution of $\left\{y_{i, t}\right\}$. We assume the following conditions.

Assumption 4 (i) $\varepsilon_{i, t} \mid\left(\left\{y_{i, s}\right\}_{s \leq t-1}, \mu_{i}\right) \sim i . i . d . \mathcal{N}\left(0, \sigma^{2}\right)$ for all $i$ and $t$, where $0<\sigma^{2}<$ $\infty$. (ii) For given $p_{0}, \sum_{j=1}^{p_{0}}\left|\alpha_{p_{0} j}\right|<\infty$ and all roots of the characteristic equation 1 $\sum_{j=1}^{p_{0}} \alpha_{p_{0} j} z^{j}=0$ lie outside the unit circle. (iii) $n^{-1} \sum_{i=1}^{n} \mu_{i}^{2}=O_{p}(1)$.

In Assumption 4-(i), we assume that the higher order lags of $y_{i, t}$ capture all the persistence, the error term is serially uncorrelated, and there is no cross sectional dependence in $\varepsilon_{i, t}$. Normality is assumed for analytic convenience, which is common in the model selection literature.

To develop a lag order selection criterion, we first obtain the maximum modified profile likelihood estimators in a Gaussian panel $A R(p)$ regression, $\widetilde{\alpha}(p)=\left(\widetilde{\alpha}_{p 1}, \cdots, \widetilde{\alpha}_{p p}\right)$ and $\widetilde{\sigma}^{2}(p)$, using the truncated sample $\left(y_{i, \bar{p}+1}, \cdots, y_{i, T}\right)$ for each $i$, where $\bar{p} \geq p_{0}$ is the maximum $A R$ lag considered. We define $y_{i, t}^{W}=y_{i, t}-\bar{T}^{-1} \sum_{s=\bar{p}+1}^{T} y_{i, s}$ as the within-transformed observation and $X_{i, t}^{W}(p)=\left(y_{i, t-1}^{W}, \cdots, y_{i, t-j}^{W}\right)^{\prime}$, where $\bar{T}=T-\bar{p}$ is the number of truncated time series

\footnotetext{
${ }^{9}$ When we are particularly interested in relatively short panels, it is reasonable to assume the true lag order $p_{0}$ to be finite. When the time series sample $T$ is longer and we allow $T \rightarrow \infty$, we can consider an approximate $A R\left(p_{T}\right)$ model with $p_{T} \rightarrow \infty$ as $T \rightarrow \infty$ with further rate conditions (e.g., $p_{T}^{3} / T \rightarrow 0$ ). When we allow for an underlying $A R(\infty)$ process, the lag order selection problem becomes one of choosing the best $A R(p)$ model to approximate the $A R(\infty)$ process.
} 
observations. Note that within-transformation corresponds to maximizing out the fixed effects $\mu_{i}$ 's in MLE (i.e., forming the profile likelihood). Using the expression of $M_{i}(\cdot)$ in (17), it can be derived that

$$
\begin{aligned}
\widetilde{\alpha}(p) & =\left[\sum_{i=1}^{n} \sum_{t=\bar{p}+1}^{T} X_{i, t}^{W}(p) X_{i, t}^{W}(p)^{\prime}+\sum_{i=1}^{n} \sum_{j=-m}^{m} \frac{K_{j}}{\bar{T}} \sum_{t=\max \{\bar{p}+1, \bar{p}+j+1\}}^{\min \{T, T+j\}} X_{i, t}^{W}(p) X_{i, t-j}^{W}(p)^{\prime}\right]^{-1} \\
& \times\left[\sum_{i=1}^{n} \sum_{t=\bar{p}+1}^{T} X_{i, t}^{W}(p) y_{i, t}^{W}+\frac{1}{2} \sum_{i=1}^{n} \sum_{j=-m}^{m} \frac{K_{j}}{\bar{T}} \sum_{t=\max \{\bar{p}+1, \bar{p}+j+1\}}^{\min \{T, T+j\}}\left\{X_{i, t}^{W}(p) y_{i, t-j}^{W}+X_{i, t-j}^{W}(p) y_{i, t}^{W}\right\}\right]
\end{aligned}
$$

and

$$
\tilde{\sigma}^{2}(p)=\frac{1}{n \bar{T}} \sum_{i=1}^{n} \sum_{t=\bar{p}+1}^{T}\left(\widetilde{\varepsilon}_{i, t}^{W}(p)\right)^{2}+\frac{1}{n \bar{T}} \sum_{i=1}^{n} \sum_{j=-m}^{m} \frac{K_{j}}{\bar{T}} \sum_{t=\max \{\bar{p}+1, \bar{p}+j+1\}}^{\min \{T, T+j\}} \widetilde{\varepsilon}_{i, t}^{W}(p) \widetilde{\varepsilon}_{i, t-j}^{W}(p),
$$

where $\widetilde{\varepsilon}_{i, t}^{W}(p)=y_{i, t}^{W}-\sum_{j=1}^{p} \widetilde{\alpha}_{p j} y_{i, t-j}^{W}$. As discussed in Section 2.3, $\widetilde{\alpha}(p)$ in 36 corresponds to the bias-corrected within-group estimator and other bias-corrected estimators can be used instead. The bias-corrected variance estimator $\widetilde{\sigma}^{2}(p)$ in 37 is novel in the literature. Instead of $\widetilde{\sigma}^{2}(p)$, the normal procedure relies on

$$
\widehat{\sigma}^{2}(p)=\frac{1}{n \bar{T}} \sum_{i=1}^{n} \sum_{t=\bar{p}+1}^{T}\left(\widetilde{\varepsilon}_{i, t}^{W}(p)\right)^{2}
$$

which is the ML estimator of $\sigma^{2}(p)$ without bias correction, where the difference between $\widetilde{\sigma}^{2}(p)$ and $\widehat{\sigma}^{2}(p)$ is of $O_{p}\left(\bar{T}^{-1}\right)$. If we denote the ratio of the long-run variance estimator to the variance estimator of $\widetilde{\varepsilon}_{i, t}^{W}(p)$ as

$$
R(p)=\frac{1}{n \widehat{\sigma}^{2}(p)} \sum_{i=1}^{n} \sum_{j=-m}^{m} \frac{K_{j}}{\bar{T}} \sum_{t=\max \{\bar{p}+1, \bar{p}+j+1\}}^{\min \{T, T+j\}} \widetilde{\varepsilon}_{i, t}^{W}(p) \widetilde{\varepsilon}_{i, t-j}^{W}(p),
$$

then

$$
\frac{2}{n} \sum_{i=1}^{n} M_{i}\left(\widetilde{\alpha}(p), \widetilde{\sigma}^{2}(p)\right)=\frac{\widehat{\sigma}^{2}(p)}{\widetilde{\sigma}^{2}(p)} \times R(p)
$$


from (17), and

$$
\widetilde{\sigma}^{2}(p)=\widehat{\sigma}^{2}(p)\left\{1+\frac{R(p)}{\bar{T}}\right\}
$$

from (37), where $R(p)=O_{p}(1)$. In addition, ignoring a constant term, we have

$$
-\frac{2}{n \bar{T}} \sum_{i=1}^{n} \sum_{t=\bar{p}+1}^{T} \log f_{i t}^{P}\left(\widetilde{\alpha}(p), \widetilde{\sigma}^{2}(p)\right)=\log \widetilde{\sigma}^{2}(p)+\frac{\widehat{\sigma}^{2}(p)}{\widetilde{\sigma}^{2}(p)}
$$

In this case, therefore, from (34), a new lag order selection criterion can be obtained as

$$
\begin{aligned}
L I C(p) & =-\frac{2}{n \bar{T}} \sum_{i=1}^{n} \sum_{t=\bar{p}+1}^{T} \log f_{i t}^{P}\left(\widetilde{\alpha}(p), \widetilde{\sigma}^{2}(p)\right)+\frac{2}{n \bar{T}} \sum_{i=1}^{n} M_{i}\left(\widetilde{\alpha}(p), \widetilde{\sigma}^{2}(p)\right)+\frac{h(n \bar{T})}{n \bar{T}} p \\
& =\left\{\log \widetilde{\sigma}^{2}(p)+\frac{\widehat{\sigma}^{2}(p)}{\widetilde{\sigma}^{2}(p)}\right\}+\left(\frac{\widehat{\sigma}^{2}(p)}{\widetilde{\sigma}^{2}(p)}\right) \frac{R(p)}{\bar{T}}+\frac{h(n \bar{T})}{n \bar{T}} p \\
& =\log \left(\widehat{\sigma}^{2}(p)\left\{1+\frac{R(p)}{\bar{T}}\right\}\right)+\left(\frac{\widehat{\sigma}^{2}(p)}{\widehat{\sigma}^{2}(p)\{1+(R(p) / \bar{T}\}}\right)\left\{1+\frac{R(p)}{\bar{T}}\right\}+\frac{h(n \bar{T})}{n \bar{T}} p
\end{aligned}
$$

where the last equality is from 39 . Using an expansion of $\log (1+(R(p) / \bar{T}))$, whose $O_{p}\left(1 / T^{2}\right)$ remainder term is expected to depend on $p$ in general, and by retaining only the relevant terms above, we can define the new lag order selection criterion as

$$
L I C(p)=\log \widehat{\sigma}^{2}(p)+\frac{p}{n \bar{T}}\left(h(n \bar{T})+\frac{n}{\bar{T}}\right)+\frac{1}{\bar{T}} R(p)
$$

for some positive $h(\cdot)$.

The first term in 40 indicates goodness-of-fit measured by the sum of the squared errors, which resembles the standard lag order selection case. As suggested in Han et al. (2013) we utilize a homogeneous time series sample in the construction of the residual variance estimates $\widehat{\sigma}^{2}(p)$ as 38 . The adjustment to employ a homogeneous time series sample in the residual variance estimates $\widehat{\sigma}^{2}(p)$ is important in controlling the probability of lag order overestimation and applies even in cases where there are no fixed effects, as shown in Han et al. (2013).

For the penalty terms in 40 , the first $(p \cdot h(n \bar{T}) / n \bar{T})$ is quite standard in model selection criteria and controls for degrees of freedom of the parameter of interest, thereby favoring par- 
simonious models. The rest of the penalty terms reflect the presence of nuisance parameters whose dimension is large. They are positive and add a heavier penalty to the information criterion, which will control for the over-selection probability. They are at most $O_{p}\left(T^{-1}\right)$ and their role becomes minor for large $T$, which is well expected since the incidental parameter problem is attenuated for large $T$. However, they can be quite important compared to the first penalty term particularly when $\bar{T}$ is small and $n$ is large.

\subsection{Discussions}

When the true lag order $p_{0}$ exists and is finite, the new order selection criterion 40 is consistent under a certain side condition, as shown in the following result. We define a lag order estimator $p^{*}$ to be consistent (and so the corresponding selection criterion is consistent) if it satisfies $\liminf \operatorname{in}_{n \rightarrow \infty} \mathbb{P}\left(p^{*}=p_{0}\right)=1$

Theorem 5 Under Assumption 4, if $n / \bar{T} \rightarrow \kappa \in(0, \infty)$ as $n, \bar{T} \rightarrow \infty$, then $L I C(p)$ is a consistent lag order selection criterion when $p_{0} \leq \bar{p}<\infty$, provided that $h(n \bar{T})$ satisfies $h(n \bar{T}) / n \bar{T} \rightarrow 0$ and $h(n \bar{T}) \rightarrow \infty$ as $n \bar{T} \rightarrow \infty$.

As discussed above, examples of $h(n \bar{T})$ for consistent criteria are $\log n \bar{T}$ and $\omega \log \log n \bar{T}$ for some $\omega \geq 2$, where the first is a $B I C$ type penalty term and the second is a $H Q$ type penalty term. Note that consistency holds even when $n / \bar{T} \rightarrow 0$. For example, when $T \rightarrow \infty$ but $n$ is fixed, $L I C(p)$ reduces to the standard lag order selection criterion in the time series context by retaining only the relevant terms (e.g., BIC: $\left.\log \widehat{\sigma}^{2}(p)+p \log \bar{T} / \bar{T}\right)$.

Theorem 5 does not provide analytical evidence explaining why the new lag order selection criteria work better than standard criteria such as $I C(p)=\log \widehat{\sigma}^{2}(p)+p(h(n \bar{T}) / n \bar{T})$. Note that this standard criterion $I C(p)$ is already modified to apply to a truncated sample as suggested by Han et al. (2013), so it is also expected to be consistent with a suitable choice of $h(n \bar{T}) \rightarrow \infty$. It can be conjectured that the under-selection probability vanishes exponentially

\footnotetext{
${ }^{10}$ This definition is somewhat different from the usual defintion of consistency but is equivalent for integer valued random variables. The lag estimator $p^{*}$ is strongly consistent if $\mathbb{P}\left(\lim _{n, \bar{T} \rightarrow \infty} p^{*}=p_{0}\right)=1$. It is known that in the standard time series context, $B I C$ and properly defined $P I C$ are strongly consistent criteria; $H Q$ is weakly consistent but not strongly consistent; and other order selection criteria, such as the final prediction error $(F P E)$ and $A I C$ are not consistent for finite $p_{0}$.
} 
fast for both cases (provided that $h(n \bar{T}) / n \bar{T} \rightarrow 0$ and $\bar{T} \rightarrow \infty$ ) similarly as Guyon and Yao (1999), while their over-selection probabilities decrease at different rates depending on the magnitude of the penalty term. Therefore, the observed improvement in correct selection probability of the new lag order selection criterion comes from reduction in the over-selection probability. The following corollary states that the over-selection probability is reduced asymptotically by modifying the penalty term as in the new lag order selection criterion given in 40 .

Corollary 6 Suppose the conditions in Theorem 5 hold. For some finite positive integer $\bar{p}$, if we let $p^{* *}=\arg \min _{0 \leq p \leq \bar{p}} I C(p)$ with $I C(p)=\log \widehat{\sigma}^{2}(p)+p(h(n \bar{T}) / n \bar{T})$ and $p^{*}=$ $\arg \min _{0 \leq p \leq \bar{p}} L I C(p)$, then $\lim \sup _{n, \bar{T} \rightarrow \infty} \mathbb{P}\left(p^{* *}>p_{0}\right) \geq \lim \sup _{n, \bar{T} \rightarrow \infty} \mathbb{P}\left(p^{*}>p_{0}\right)$.

Finally, we study the finite sample performance of the new lag order selection criterion and compare it with conventional time series model selection methods. We define the two most commonly used information criteria, which use the pooled information as $I C(p)$ in Corollary 6 .

$$
\begin{aligned}
& A I C(p)=\log \widehat{\sigma}^{2}(p)+\frac{2}{n \bar{T}} p, \\
& B I C(p)=\log \widehat{\sigma}^{2}(p)+\frac{\log n \bar{T}}{n \bar{T}} p,
\end{aligned}
$$

where $\widehat{\sigma}^{2}(p)$ is defined as 38 using the truncated uniform time series sample following Han et al. (2013). Preliminary simulation results show that constructing penalty terms using the parameter count $p+n$ (i.e., including fixed effect parameters) too heavily penalizes the criteria so that they yield high under-selection probabilities. We thus only count the number of parameters as $p$ in defining the information criteria above. For the new criteria, we consider the following forms suggested in (40):

$$
\begin{aligned}
& L I C^{A I C}(p)=\log \widehat{\sigma}^{2}(p)+\frac{p}{n \bar{T}}\left(2+\frac{n}{\bar{T}}\right)+\frac{1}{\bar{T}} R(p), \\
& L I C^{B I C}(p)=\log \widehat{\sigma}^{2}(p)+\frac{p}{n \bar{T}}\left(\log n \bar{T}+\frac{n}{\bar{T}}\right)+\frac{1}{\bar{T}} R(p) .
\end{aligned}
$$


We generate $A R(3)$ dynamic panel processes of the form $y_{i, t}=\mu_{i}+\sum_{j=1}^{3} \alpha_{3 j} y_{i, t-j}+\varepsilon_{i, t}$ for $i=1,2, \cdots, n$ and $t=1,2, \cdots, T$, where $\alpha_{3 j}=0.15$ for all $j=1,2,3$. This design is analogous to the one used in the simulation study of Han et al. (2013). All the autoregressive coefficients have the same value so that the lagged terms are equally important. We also consider the case of negative autocorrelations with $\alpha_{3 j}=-0.15$ for all $j=1,2,3$, but the simulation results are almost the same. We consider 64 different cases by combining different sample sizes of $n=100,200,300, \cdots, 800$ and $T=25,30,35, \cdots, 60$. Fixed effects $\mu_{i}$ are randomly drawn from $\mathcal{U}(-0.5,0.5)$ and $\varepsilon_{i, t}$ from $\mathcal{N}(0,1)$. We use the bias corrected withingroup estimators (e.g., Lee (2012)) for the $\widetilde{\alpha}_{p j}$ and replicate the entire procedure 1000 times to compare the performance of different order selection criteria. For each case, we choose the optimal lag order $p^{*}$ to minimize the criteria above, where we search over lag orders from 1 to 7 (i.e., $\bar{p}=7$ ). The simulation results are provided in Figures 1 to 3, which present the correct-selection, over-selection, and under-selection probabilities of each case, respectively.

[Figures 1, 2, 3 are about here]

Figure 1 shows clearly that the new lag order selection criteria $L I C^{A I C}$ and $L I C^{B I C}$ perform much better than the common criteria $A I C$ and $B I C$. Though not consistent, the correct selection probability of $L I C^{A I C}$ reaches a level close to unity ${ }^{11}$ With the new criteria the correct-selection probability improves rapidly with $T$ and does so uniformly over $n$. From Figures 2 and 3 it is evident that the improvement comes from the reduction in the over-selection probability. Since we impose a heavier penalty, however, the under-selection probability is high for very small $T$, which corresponds to the well-known property of $B I C$ in a pure time series setup.

By comparison Figure 1 shows that the common criteria perform poorly with large $n$, and consistency seems to hold only with very large $T$ and small $n$. From Figures 2 and 3, such poor performance is due to the high over-selection probability discussed in the previous

\footnotetext{
${ }^{11}$ We conjecture that $L I C^{A I C}$ is asymptotically optimal (i.e., $\operatorname{plim}_{n, \bar{T} \rightarrow \infty}\left[L I C^{A I C}\left(p^{*}\right) / \inf _{p \geq 0} L I C^{A I C}(p)\right]=$ 1 , where $p^{*}$ is the lag order estimator from $L I C^{A I C}(p)$, e.g., Shibata (1980), Li (1987), Yang (2005)) if the true data generating model is $A R(\infty)$ with finite $\sigma^{2}$.
} 
subsection. Even $B I C$ tends to overfit the order in dynamic panel models, where the overselection probability increases quite fast with $n$. This finding is contrary to the well known property that $B I C$ normally underfits lag order in a pure time series setup. In addition, since $B I C$ is formulated here in the modified form developed by Han et al. (2013) with a uniformly truncated sample (to ensure consistency), it is apparent that this modified criterion seems to require large $T$ to perform well when the dynamic panel model includes individual fixed effects.

\section{Concluding Remarks}

It is not uncommon in empirical work for a subset of parameters to be the central interest. In such cases, the nuisance parameters account for aspects of the model that are not of immediate concern but are nonetheless needed for realistic statistical modeling. Particularly when the dimension of the nuisance parameter space is large, dealing adequately with nuisance parameters is important for valid inference. As we demonstrate, model selection also needs to account for the presence of nuisance parameters to obtain correct model specification. The approach adopted in the present paper is to deal with nuisance parameters using either the profile likelihood (for AIC-type selectors) or integrated likelihood (for BIC-type selectors). The result is a new model selection criterion that can be used in the presence of nuisance parameters. The new penalty term in the selector is data-dependent and properly controls for model complexity.

Incidental parameters form a subset of parameters whose estimators typically have slower rates of convergence than those of the primary parameters under dual index asymptotics. We may therefore view the present paper as addressing a special case of a more general question: model selection involving a sub-set of parameters when the remaining parameters are estimable only at a slower rate of convergence than the primary parameters. Semiparametric models come within the same framework when we consider the nonparametric component as

an infinite dimensional nuisance parameter using a similar approach to Severini and Wong (1992), particularly when some orthogonality condition (e.g., Andrews (1994), Newey (1994)) 
is violated.

For the particular problem of lag order selection in panel autoregression, Han et al. (2013) recently showed that the conventional BIC selector is inconsistent even in a panel model without fixed effects. The analysis in Han et al. (2013) reveals that dual index asymptotics typically induce order overestimation (with an asymptotic probability as high as 50\%) in lag order selectors. The heuristic reason for the overestimation is that residual variance estimates in panel models with lag orders that exceed the true value will involve fewer innovations than the residual variance estimate obtained from the true dynamic specification. Cross section averaging then produces $O(n)$ such differences (which after normalization contribute $O(1 / \sqrt{n} T)$ rather than $O(1 / n T)$ to the fit component of the selector) and these components end up dominating the standard BIC penalty, thereby blinding BIC to the overspecification. Modifications to BIC that are explored in Han et al. (2013) involve increasing the penalty, as we have done in the present paper to attenuate overspecification, and truncating the time series sample so that a common sample is used for the residual variance calculation. With these modifications, the BIC criterion is a consistent lag order selector in panel autoregression with fixed effects. 


\section{Appendix: Proofs}

Proof of Lemma 1 The result follows immediately since

$$
\begin{aligned}
D_{P}\left(g_{i}(\cdot) \| f\left(\cdot ; \psi, \lambda_{i}\right) ; \psi\right)= & \int \log g_{i}(z) d G_{i}(z)-\int \log f\left(z ; \psi, \lambda_{i}(\psi)\right) d G_{i}(z) \\
= & \int \log g_{i}(z) d G_{i}(z)-\int \log f\left(z ; \psi, \widehat{\lambda}_{i}(\psi)\right) d G_{i}(z) \\
& +\int \log \left(\frac{f\left(z ; \psi, \widehat{\lambda}_{i}(\psi)\right)}{f\left(z ; \psi, \lambda_{i}(\psi)\right)}\right) d G_{i}(z)
\end{aligned}
$$

and

$$
\begin{aligned}
& \int \log \left(\frac{f\left(z ; \psi, \widehat{\lambda}_{i}(\psi)\right)}{f\left(z ; \psi, \lambda_{i}(\psi)\right)}\right) d G_{i}(z) \\
= & \int \frac{1}{T} \sum_{t=1}^{T}\left\{\log f\left(z_{i, t} ; \psi, \widehat{\lambda}_{i}(\psi)\right)-\log f\left(z_{i, t} ; \psi, \lambda_{i}(\psi)\right)\right\} d G_{i}\left(z_{i, t}\right)=\delta_{i}(\psi)
\end{aligned}
$$

by stationarity. Furthermore, from $(16)$ and $(17)$, it can be seen that

$$
\mathbb{E}_{G_{i}}\left[\delta_{i}\left(\psi_{0}\right)-\frac{M_{i}\left(\psi_{0}\right)}{T}\right]=\mathbb{E}_{G_{i}}\left[\ell_{i}^{P}\left(\psi_{0}\right)-\ell_{i}\left(\psi_{0}, \lambda_{i}\left(\psi_{0}\right)\right)-\frac{M_{i}\left(\psi_{0}\right)}{T}\right]=O\left(\frac{1}{T^{3 / 2}}\right) .
$$

Proof of Theorem 2 For each $i$, define $G_{i}(\cdot ; \epsilon)=G_{i}(\cdot)+\epsilon\left(\widehat{G}_{i}(\cdot)-G_{i}(\cdot)\right)$ for some $\epsilon \in[0,1]$. $G(\cdot ; \epsilon), G(\cdot)$ and $\widehat{G}(\cdot)$ denote the collection of the marginal distributions (i.e., $G(Z ; \epsilon)=$ $\left(G_{1}\left(z_{1} ; \epsilon\right), \cdots, G_{n}\left(z_{n} ; \epsilon\right)\right)$ with $Z=\left(z_{1}, \cdots, z_{n}\right)^{\prime}$ and similarly for the others). We also use notations $G_{i}$ and $\widehat{G}_{i}$ instead of $G_{i}(\cdot)$ and $\widehat{G}_{i}(\cdot)$ when there is no risk of confusion. We denote the likelihood function evaluated at the observation $z_{i, t}$ as $f_{i t}\left(z ; \psi, \lambda_{i}\right)$ or $f_{i t}\left(z_{i, t} ; \psi, \lambda_{i}\right)$ as needed.

For a fixed $\epsilon$ and an $r$-dimensional regular function $H$ that is second-order Hadamard differentiable at $G$, let $\psi(\epsilon)=H(G(\cdot ; \epsilon))$ be the solution of

$$
\frac{1}{n} \sum_{i=1}^{n} \int \frac{\partial}{\partial \psi} Q_{i t}(z ; \epsilon) d G_{i}(z ; \epsilon)=0,
$$


where

$$
Q_{i t}(z ; \epsilon)=\log f_{i t}\left(z ; \psi(\epsilon), \lambda_{i}(\epsilon)\right)-\frac{1}{T} \mu_{i}(\epsilon) .
$$

$\lambda_{i}(\epsilon)=\lambda_{i}\left(\psi(\epsilon) ; G_{i}(z ; \epsilon)\right)$ is the solution of $\int\left[\partial \log f_{i t}\left(z ; \psi(\epsilon), \lambda_{i}(\epsilon)\right) / \partial \lambda_{i}\right] d G_{i}(z ; \epsilon)=0$ for each $i$ so that

$$
\lambda_{i}(\epsilon)= \begin{cases}\lambda_{i}\left(\psi(0) ; G_{i}\right)=\lambda_{i}(\psi(0)) & \text { if } \epsilon=0 \\ \lambda_{i}\left(\psi(1) ; \widehat{G}_{i}\right)=\widehat{\lambda}_{i}(\psi(1)) & \text { if } \epsilon=1,\end{cases}
$$

and $\mu_{i}(\epsilon)=\epsilon M_{i}(\psi(\epsilon))$ yielding

$$
\mu_{i}(\epsilon)= \begin{cases}\mu_{i}(0)=0 & \text { if } \epsilon=0 \\ \mu_{i}(1)=M_{i}(\psi(1)) & \text { if } \epsilon=1,\end{cases}
$$

where $\hat{\lambda}_{i}(\psi), \lambda_{i}(\psi)$ and $M_{i}(\psi)$ are defined as (3), (8) and (5), respectively. It follows that $\psi(0)=H(G)=\psi_{0}$ and $\psi(1)=H(\widehat{G})=\widetilde{\psi}$ by construction. Note that $\psi_{0}$ minimizes $\lim _{n \rightarrow \infty} n^{-1} \sum_{i=1}^{n} \int Q_{i t}(z ; 0) G_{i}(z)=0$ as defined in $(9)$ and thus $\psi_{0}$ also be a solution of (A.1) when $\epsilon=0$.

Denote each element of $\psi(\cdot)$ and $H(\cdot)$ as $\psi_{[l]}(\cdot)$ and $H_{[l]}(\cdot)$ for $l=1,2, \cdots, r$, respectively. Then, for $n, T \rightarrow \infty$ satisfying $n / T \rightarrow \kappa \in(0, \infty)$, the functional Taylor series expansion of $\psi_{[l]}(1)$ about $\psi_{[l]}(0)$ for each $l$ can be obtained as (e.g., Chapter 6.2 in Serfling (1980), Konishi and Kitagawa (1996))

$$
\begin{aligned}
\psi_{[l]}(1)-\psi_{[l]}(0) & =H_{[l]}(\widehat{G})-H_{[l]}(G) \\
& =d_{1} H_{[l]}(G ; \widehat{G}-G)+\frac{1}{2} d_{2} H_{[l]}(G ; \widehat{G}-G)+o_{p}\left(\frac{1}{n T}\right) \\
& =\frac{1}{n T} \sum_{i=1}^{n} \sum_{t=1}^{T} H_{[l]}^{(1)}\left(z_{i, t} ; G\right)+\frac{1}{2 n^{2} T^{2}} \sum_{i, j=1}^{n} \sum_{t, s=1}^{T} H_{[l]}^{(2)}\left(z_{i, t}, z_{j, s} ; G\right)+o_{p}\left(\frac{1}{n T}\right)
\end{aligned}
$$

where $d_{1} H_{[l]}(G ; \widehat{G}-G)=\lim _{\epsilon \rightarrow 0+} \epsilon^{-1}\left\{H_{[l]}(G(\epsilon))-H_{[l]}(G)\right\}$ is the Gâteaux differential of $H_{[l]}$ at $G$ in the direction of $\widehat{G} ; d_{k} H_{[l]}(G ; \widehat{G}-G)=d^{k} H_{[l]}(G(\epsilon)) /\left.d \epsilon^{k}\right|_{\epsilon=0+}$ provided limit exists; $H_{[l]}^{(k)}$ is defined as $d^{k} H_{[l]}(G(\epsilon)) / d \epsilon^{k}=\int \cdots \int H_{[l]}^{(k)}\left(z^{1}, \cdots, z^{k} ; G\right) \prod_{a=1}^{k} d\left(\widehat{G}\left(z^{a}\right)-G\left(z^{a}\right)\right)$ at $\epsilon=0$. Note that $H^{(1)}(z ; G)=\left(H_{[1]}^{(1)}(z ; G), \cdots, H_{[r]}^{(1)}(z ; G)\right)^{\prime}$ is the influence function of $H$ at $G$ (e.g., Huber (1981)) satisfying $\int H^{(1)}(z ; G) d G(z)=0$. 
Similar to Hahn and Kuersteiner (2011), by differentiating (A.1) with respect to $\epsilon$, we have

$$
\begin{aligned}
0= & \frac{1}{n} \sum_{i=1}^{n} \int \frac{\partial^{2}}{\partial \psi \partial \psi^{\prime}} Q_{i t}(z ; \epsilon) d G_{i}(z ; \epsilon) \times d_{1} H(G ; \widehat{G}-G) \\
& +\frac{1}{n} \sum_{i=1}^{n} \int \frac{\partial^{2}}{\partial \psi \partial \lambda_{i}} Q_{i t}(z ; \epsilon) d G_{i}(z ; \epsilon) \times \frac{\partial}{\partial \epsilon} \lambda_{i}(\epsilon) \\
& +\frac{1}{n} \sum_{i=1}^{n} \int \frac{\partial}{\partial \psi} Q_{i t}(z ; \epsilon) d\left(\widehat{G}_{i}(z)-G_{i}(z)\right),
\end{aligned}
$$

where $d_{1} H(G ; \widehat{G}-G)=\left(d_{1} H_{[1]}(G ; \widehat{G}-G), \cdots, d_{1} H_{[r]}(G ; \widehat{G}-G)\right)^{\prime}$, and by evaluating this result at $\epsilon=0$ we find

$$
\begin{aligned}
d_{1} H(G ; \widehat{G}-G)= & \left(-\frac{1}{n} \sum_{i=1}^{n} \int \frac{\partial^{2} \log f_{i t}\left(z ; \psi(0), \lambda_{i}(\psi(0))\right)}{\partial \psi \partial \psi^{\prime}} d G_{i}(z)\right)^{-1} \\
& \times \frac{1}{n} \sum_{i=1}^{n} \int \frac{\partial \log f_{i t}\left(z ; \psi(0), \lambda_{i}(\psi(0))\right)}{\partial \psi} d \widehat{G}_{i}(z)
\end{aligned}
$$

since $\int\left[\partial \log f_{i t}\left(z ; \psi(0), \lambda_{i}(\psi(0))\right) / \partial \psi\right] d G_{i}(z)=0$ and $\int\left[\partial^{2} \log f_{i t}\left(z ; \psi(0), \lambda_{i}(\psi(0))\right) / \partial \psi \partial \lambda_{i}\right] d G_{i}(z)=$ 0. We thus obtain the explicit expression of $H^{(1)}\left(z_{i, t} ; G\right)$ as (e.g., Withers 1983 , Konishi and Kitagawa (1996) 12

$$
\begin{aligned}
H^{(1)}\left(z_{i, t} ; G\right)= & \left(-\left.\frac{1}{n} \sum_{i=1}^{n} \int \frac{\partial^{2} \log f_{i t}\left(z ; \psi, \lambda_{i}(\psi)\right)}{\partial \psi \partial \psi^{\prime}}\right|_{\psi=\psi(0)} d G_{i}(z)\right)^{-1} \\
& \times\left.\frac{\partial \log f_{i t}\left(z_{i, t} ; \psi, \lambda_{i}(\psi)\right)}{\partial \psi}\right|_{\psi=\psi(0)}
\end{aligned}
$$

We do not find $H^{(2)}\left(z_{i, t}, z_{j, s} ; G\right)$ in details since we only need an expression of $H^{(1)}\left(z_{i, t} ; G\right)$ to derive the main result.

Similar to Theorem 2.1 of Konishi and Kitagawa $(1996)$, by expanding $f_{i t}^{P}(z ; \widetilde{\psi})=$ $f_{i t}\left(z ; \psi(1), \widehat{\lambda}_{i}(\psi(1))\right)$ around $\psi(0)=\psi_{0}$ for given $i$ and $t$ and combining the result in A.2,

\footnotetext{
${ }^{12}$ From $\mathrm{A} .2$, it also shows that $\widehat{\psi}_{M}$ is $\sqrt{n T}$-consistent to $\psi_{0}$ since $(n T)^{-1} \sum_{i, j=1}^{n} \sum_{t, s=1}^{T} H^{(2)}\left(z_{i, t}, z_{j, s} ; G\right)=$ $O_{p}(1 / n T)$ and $(n T)^{-1 / 2} \sum_{i=1}^{n} \sum_{t=1}^{T} H^{(1)}\left(z_{i, t} ; G_{i}\right)$ is asymptotically normal with mean zero and variance $\lim _{n, T \rightarrow \infty}(n T)^{-1} \sum_{i=1}^{n} \int \sum_{t, s=1}^{T} H^{(1)}\left(z_{i, t} ; G_{i}\right) H^{(1)}\left(z_{i, s} ; G_{i}\right)^{\prime} d G_{i}<\infty$.
} 
we then have a stochastic expansion as

$$
\begin{aligned}
& \int \log f_{i t}^{P}(z ; \widetilde{\psi}) d G_{i}(z) \\
= & \int \log f_{i t}\left(z ; \psi_{0}, \widehat{\lambda}_{i}\left(\psi_{0}\right)\right) d G_{i}(z) \\
& +\frac{1}{n T} \sum_{j=1}^{n} \sum_{s=1}^{T} \sum_{l=1}^{r} H_{[l]}^{(1)}\left(z_{j, s} ; G\right) \int \frac{\partial \log f_{i t}\left(z ; \psi_{0}, \widehat{\lambda}_{i}\left(\psi_{0}\right)\right)}{\partial \psi_{[l]}} d G_{i}(z) \\
& +\frac{1}{2 n^{2} T^{2}} \sum_{j, j^{\prime}=1}^{n} \sum_{s, s^{\prime}=1}^{T}\left\{\sum_{l=1}^{r} H_{[l]}^{(2)}\left(z_{j, s}, z_{j^{\prime}, s^{\prime}} ; G\right) \int \frac{\partial \log f_{i t}\left(z ; \psi_{0}, \widehat{\lambda}_{i}\left(\psi_{0}\right)\right)}{\partial \psi_{[l]}} d G_{i}(z)\right. \\
& \left.+\sum_{l, l^{\prime}=1}^{r} H_{[l]}^{(1)}\left(z_{j, s} ; G\right) H_{\left[l^{\prime}\right]}^{(1)}\left(z_{j^{\prime}, s^{\prime}} ; G\right) \int \frac{\partial^{2} \log f_{i t}\left(z ; \psi_{0}, \widehat{\lambda}_{i}\left(\psi_{0}\right)\right)}{\partial \psi_{[l]} \partial \psi_{\left[l^{\prime}\right]}} d G_{i}(z)\right\}+o_{p}\left(\frac{1}{n T}\right) .
\end{aligned}
$$

Using $\mathbb{E}[\cdot]$ to signify expectation with respect to the joint distribution of $\left(G_{1}, \cdots, G_{n}\right)$, we have

$$
\begin{aligned}
& \mathbb{E}\left[\frac{1}{n T} \sum_{i=1}^{n} \sum_{t=1}^{T} \int \log f_{i t}^{P}(z ; \widetilde{\psi}) d G_{i}(z)\right] \\
= & \frac{1}{n T} \sum_{i=1}^{n} \sum_{t=1}^{T} \int \log f_{i t}\left(z ; \psi(0), \widehat{\lambda}_{i}(\psi(0))\right) d G_{i}(z)+V_{n, T}+o\left(\frac{1}{n T}\right)
\end{aligned}
$$

since the expectation of the second term in A.5 becomes zero for $\int H_{[l]}^{(1)}\left(z_{j, s} ; G\right) d G_{j}(z)=0$ for all $j$ and $l$. Note that $V_{n, T}$ corresponds to the expectation of the third term in A.5, where

$$
\begin{aligned}
V_{n, T}= & \frac{1}{2 n^{3} T^{3}} \sum_{i, j=1}^{n} \sum_{t, s, s^{\prime}=1}^{T}\left\{\sum_{l=1}^{r} \mathbb{E}_{G_{j}}\left[H_{[l]}^{(2)}\left(z_{j, s}, z_{j, s^{\prime}} ; G\right)\right] \int \frac{\partial \log f_{i t}\left(z ; \psi_{0}, \widehat{\lambda}_{i}\left(\psi_{0}\right)\right)}{\partial \psi_{[l]}} d G_{i}(z)\right. \\
& \left.+\sum_{l, l^{\prime}=1}^{r} \mathbb{E}_{G_{j}}\left[H_{[l]}^{(1)}\left(z_{j, s} ; G\right) H_{\left[l^{\prime}\right]}^{(1)}\left(z_{j, s^{\prime}} ; G\right)\right] \int \frac{\partial^{2} \log f_{i t}\left(z ; \psi_{0}, \widehat{\lambda}_{i}\left(\psi_{0}\right)\right)}{\partial \psi_{[l]} \partial \psi_{\left[l^{\prime}\right]}} d G_{i}(z)\right\},
\end{aligned}
$$


which is nonzero only for the case with $j=j^{\prime}$. Similarly,

$$
\begin{aligned}
& \int \log f_{i t}^{P}(z ; \widetilde{\psi}) d \widehat{G}_{i}(z) \\
= & \frac{1}{T} \sum_{t=1}^{T} \log f_{i t}\left(z_{i, t} ; \psi_{0}, \widehat{\lambda}_{i}\left(\psi_{0}\right)\right) \\
& +\frac{1}{n T^{2}} \sum_{j=1}^{n} \sum_{t, s=1}^{T} \sum_{l=1}^{r} H_{[l]}^{(1)}\left(z_{j, s} ; G\right) \frac{\partial \log f_{i t}\left(z_{i, t} ; \psi_{0}, \widehat{\lambda}_{i}\left(\psi_{0}\right)\right)}{\partial \psi_{[l]}} \\
& +\frac{1}{2 n^{2} T^{3}} \sum_{j, j^{\prime}=1}^{n} \sum_{t, s, s^{\prime}=1}^{T}\left\{\sum_{l=1}^{r} H_{[l]}^{(2)}\left(z_{j, s}, z_{j^{\prime}, s^{\prime}} ; G\right) \frac{\partial \log f_{i t}\left(z_{i, t} ; \psi_{0}, \widehat{\lambda}_{i}\left(\psi_{0}\right)\right)}{\partial \psi_{[l]}}\right. \\
& \left.+\sum_{l, l^{\prime}=1}^{r} H_{[l]}^{(1)}\left(z_{j, s} ; G\right) H_{\left[l^{\prime}\right]}^{(1)}\left(z_{j^{\prime}, s^{\prime}} ; G\right) \frac{\partial^{2} \log f_{i t}\left(z_{i, t} ; \psi_{0}, \widehat{\lambda}_{i}\left(\psi_{0}\right)\right)}{\partial \psi_{[l]} \partial \psi_{\left[l^{\prime}\right]}}\right\}+o_{p}\left(\frac{1}{n T}\right)
\end{aligned}
$$

and by stationarity over $t$

$$
\begin{aligned}
& \mathbb{E}\left[\frac{1}{n T} \sum_{i=1}^{n} \sum_{t=1}^{T} \int \log f_{i t}^{P}(z ; \widetilde{\psi}) d \widehat{G}_{i}(z)\right] \\
= & \frac{1}{n T} \sum_{i=1}^{n} \sum_{t=1}^{T} \int \log f_{i t}\left(z ; \psi_{0}, \widehat{\lambda}_{i}\left(\psi_{0}\right)\right) d G_{i}(z) \\
& +\frac{1}{n^{2} T^{2}} \sum_{i=1}^{n} \sum_{t, s=1}^{T} \sum_{l=1}^{r} \int H_{[l]}^{(1)}\left(z_{i, s} ; G\right) \frac{\partial \log f_{i t}\left(z_{i, t} ; \psi_{0}, \widehat{\lambda}_{i}\left(\psi_{0}\right)\right)}{\partial \psi_{[l]}} d G_{i}(z)+V_{n, T}+o\left(\frac{1}{n T}\right),
\end{aligned}
$$

where the second term is nonzero only for the case with $i=j$, and the third term becomes $V_{n, T}$ as above. Therefore,

$$
\begin{aligned}
& \mathbb{E}\left[-\frac{1}{n T} \sum_{i=1}^{n} \sum_{t=1}^{T} \int \log f_{i t}^{P}(z ; \widetilde{\psi}) d\left(\widehat{G}_{i}(z)-G_{i}(z)\right)\right] \\
= & -\frac{1}{n^{2} T^{2}} \sum_{i=1}^{n} \int \sum_{t, s=1}^{T} \frac{\partial}{\partial \psi^{\prime}} \log f_{i t}\left(z ; \psi_{0}, \widehat{\lambda}_{i}\left(\psi_{0}\right)\right) H^{(1)}\left(z_{i, s} ; G\right) d G_{i}(z)+o\left(\frac{1}{n T}\right) \\
= & -\frac{1}{n T} \operatorname{tr}\left\{\left(-\left.\frac{1}{n} \sum_{i=1}^{n} \int \frac{\partial^{2} \log f_{i t}\left(z ; \psi, \lambda_{i}(\psi)\right)}{\partial \psi \partial \psi^{\prime}}\right|_{\psi=\psi_{0}} d G_{i}(z)\right)^{-1} \times\right. \\
& \left.\frac{1}{n} \sum_{i=1}^{n} \int\left(\left.\left.\frac{1}{T} \sum_{t, s=1}^{T} \frac{\partial \log f_{i t}\left(z ; \psi, \lambda_{i}(\psi)\right)}{\partial \psi}\right|_{\psi=\psi_{0}} \frac{\partial \log f_{i s}\left(z ; \psi, \widehat{\lambda}_{i}(\psi)\right)}{\partial \psi^{\prime}}\right|_{\psi=\psi_{0}}\right) d G_{i}(z)\right\}+o\left(\frac{1}{n T}\right)
\end{aligned}
$$

by substituting A.4), where the expression of $J(G)$ comes from stationarity over $t$. This 
result gives the expression for $B_{P}(\widetilde{\psi})$.

Proof of Corollary 3 First note that $\partial \ell_{i}\left(\psi_{0}, \lambda_{i}\left(\psi_{0}\right)\right) / \partial \psi=u_{i}^{e}$ by construction. Therefore, when $g_{i}(\cdot)$ is nested in $f\left(\cdot ; \psi, \lambda_{i}\right)$, the standard information matrix identity gives

$$
J(G)=\frac{1}{n} \sum_{i=1}^{n} \int-\frac{\partial^{2} \ell_{i}\left(\psi_{0}, \lambda_{i}\left(\psi_{0}\right)\right)}{\partial \psi \partial \psi^{\prime}} d G_{i}=\frac{1}{n} \sum_{i=1}^{n} T \int u_{i}^{e} u_{i}^{e \prime} d G_{i},
$$

where the first equality uses the stationarity over $t$. For $I(G)$, since $\partial \ell_{i}^{P}\left(\psi_{0}\right) / \partial \psi=u_{i}^{e}+$ $b_{i}\left(\psi_{0}\right)+O_{p}\left(T^{-3 / 2}\right)$ with $u_{i}^{e}=O_{p}\left(T^{-1 / 2}\right)$ and $b_{i}\left(\psi_{0}\right)=O_{p}\left(T^{-1}\right)$ from 12 , we have

$$
\begin{aligned}
I(G) & =\frac{1}{n} \sum_{i=1}^{n} T \int\left[\frac{\partial \ell_{i}\left(\psi_{0}, \lambda_{i}\left(\psi_{0}\right)\right)}{\partial \psi} \frac{\partial \ell_{i}^{P}\left(\psi_{0}\right)}{\partial \psi^{\prime}}\right] d G_{i} \\
& =\frac{1}{n} \sum_{i=1}^{n} T\left\{\int u_{i}^{e} u_{i}^{e \prime} d G_{i}+\int u_{i}^{e} b_{i}\left(\psi_{0}\right)^{\prime} d G_{i}+o\left(T^{-3 / 2}\right)\right\} \\
& =\frac{1}{n} \sum_{i=1}^{n} T\left\{\int u_{i}^{e} u_{i}^{e \prime} d G_{i}+O\left(T^{-3 / 2}\right)\right\}
\end{aligned}
$$

where the remaining term in the second equality is $o\left(T^{-3 / 2}\right)$ since $\int\left[\partial \ell_{i}\left(\psi_{0}, \lambda_{i}\left(\psi_{0}\right)\right) / \partial \psi\right] d G_{i}=$ $\int u_{i}^{e} d G_{i}=0$. Therefore, by plugging A.6) and A.7 into the expression of $B_{p}(\widetilde{\psi})$ in Theorem 2, we have

$$
B_{p}(\widetilde{\psi})=-\frac{r}{n T}+O\left(\frac{1}{n T^{3 / 2}}\right)+o\left(\frac{1}{n T}\right)=-\frac{r}{n T}+o\left(\frac{1}{n T}\right),
$$

from which the information criterion $(28)$ is obtained.

Proof of Theorem 4 The $\log$ conditional prior is given as $\log \pi_{i}\left(\lambda_{i} \mid \psi^{k}\right)=-(1 / 2) \log (2 \pi / T)+$ $(1 / 2) \log \mathbb{E}_{\widehat{G}_{i}}\left[-\partial^{2} \ell_{i}\left(\psi^{k}, \lambda_{i}\right) / \partial \lambda_{i}^{2}\right]-(1 / 2)\left(\mathbb{E}_{\widehat{G}_{i}}\left[-\partial^{2} \ell_{i}\left(\psi^{k}, \lambda_{i}\right) / \partial \lambda_{i}^{2}\right]\right)^{-1}\left(T \mathbb{E}_{\widehat{G}_{i}}\left[\partial \ell_{i}\left(\psi^{k}, \lambda_{i}\right) / \partial \lambda_{i}\right]\right)^{2}$, which is $O(1)$ as $T \rightarrow \infty$ satisfying Assumption 3-(ii). By plugging it into the approximation (30), the log posterior probability of model $\mathcal{M}^{k}$ in 29 can be written as

$$
\begin{aligned}
\log \mathcal{P}\left(\mathcal{M}^{k} \mid z\right)= & -\log g(y)+\log \phi^{k}+\log \int \exp \left(T \sum_{i=1}^{n} \ell_{i}^{I}\left(\psi^{k}\right)\right) \eta\left(\psi^{k} \mid \mathcal{M}^{k}\right) d \psi^{k} \\
= & -\log g(y)+\log \phi^{k} \\
& +\log \int \exp \left(\sum_{i=1}^{n} T\left\{\ell_{i}^{M}\left(\psi^{k}\right)+O_{p}\left(\frac{1}{T^{2}}\right)\right\}\right) \eta\left(\psi^{k} \mid \mathcal{M}^{k}\right) d \psi^{k}
\end{aligned}
$$


But Taylor expansion yields

$$
T \sum_{i=1}^{n} \ell_{i}^{M}\left(\psi^{k}\right)=T \sum_{i=1}^{n} \ell_{i}^{M}\left(\widetilde{\psi}^{k}\right)-\frac{1}{2}\left(\widetilde{\psi}^{k}-\psi^{k}\right)^{\prime}\left[n T \widehat{\mathcal{I}}\left(\widetilde{\psi}^{k}\right)\right]\left(\widetilde{\psi}^{k}-\psi^{k}\right)+o_{p}(1),
$$

where $\widetilde{\psi}^{k}$ is the modified profile ML estimator of the model $\mathcal{M}^{k}$ and

$$
\widehat{\mathcal{I}}\left(\widetilde{\psi}^{k}\right)=\frac{1}{n} \sum_{i=1}^{n} \widehat{\mathcal{I}}_{i}\left(\widetilde{\psi}^{k}\right)=-\frac{1}{n T} \sum_{i=1}^{n} \sum_{t=1}^{T} \frac{\partial \log f\left(z_{i, t} ; \widetilde{\psi}^{k}, \widehat{\lambda}_{i}\left(\widetilde{\psi}^{k}\right)\right)}{\partial \theta_{i}} \cdot \frac{\partial \log f\left(z_{i, t} ; \widetilde{\psi}^{k}, \widehat{\lambda}_{i}\left(\widetilde{\psi}^{k}\right)\right)}{\partial \theta_{i}^{\prime}}
$$

is the averaged information matrix estimator. Note that $\widetilde{\psi}^{k}-\psi^{k}=O_{p}\left((n T)^{-1 / 2}\right)$ when $n / T \rightarrow \kappa \in(0, \infty)$ and $\widehat{\mathcal{I}}\left(\widetilde{\psi}^{k}\right)=O_{p}(1)$ from Assumptions 1 and 2 . Therefore, using the uninformative flat prior $\eta\left(\psi^{k} \mid \mathcal{M}^{k}\right)=1$, Laplace approximation (e.g., Phillips $(1980,1983)$, Tierney et al. (1989)) gives

$$
\log \int \exp \left(T \sum_{i=1}^{n} \ell_{i}^{M}\left(\psi^{k}\right)\right) d \psi^{k}=T \sum_{i=1}^{n} \ell_{i}^{M}\left(\widetilde{\psi}^{k}\right)+\log \left\{(2 \pi)^{r_{k} / 2}\left|n T \widehat{\mathcal{I}}\left(\widetilde{\psi}^{k}\right)\right|^{-1 / 2}\right\}+o_{p}(1)
$$

and thus

$$
\begin{aligned}
\log \mathcal{P}\left(\mathcal{M}^{k} \mid z\right)= & -\log g(y)+\log \phi^{k}+O_{p}\left(\frac{n}{T}\right) \\
& +T \sum_{i=1}^{n} \ell_{i}^{M}\left(\widetilde{\psi}^{k}\right)+\frac{r_{k}}{2} \log 2 \pi-\frac{r_{k}}{2} \log n T-\frac{1}{2} \log \left|\widehat{\mathcal{I}}\left(\widetilde{\psi}^{k}\right)\right|+o_{p}(1),
\end{aligned}
$$

where $r_{k}=\operatorname{dim}\left(\psi^{k}\right)$. The result 32 follows by collecting terms that do not depend on $k$ and terms that are bounded as $n, T \rightarrow \infty$ as $c(z, k)=-\log g(y)+\log \phi^{k}+O_{p}(n / T)+$ $\left(r_{k} / 2\right) \log 2 \pi-(1 / 2) \log \left|\widehat{\mathcal{I}}\left(\widetilde{\psi}^{k}\right)\right|$, which is $O_{p}(1)$.

Proof of Theorem 5 Recall that the selection rule is to choose $p^{*}$ if $L I C\left(p^{*}\right)<L I C(p)$, where $0 \leq p^{*}, p \leq \bar{p}$ for some finite positive integer $\bar{p}$. We therefore need to prove that $\limsup _{n, \bar{T} \rightarrow \infty} \mathbb{P}\left[L I C\left(p^{*}\right)<L I C\left(p_{0}\right)\right]=0$ for all $p^{*} \neq p_{0}$, where $p_{0}$ is the (finite) true lag order. 
First consider the case of under-selection, $p^{*}<p_{0}$. We write

$$
\begin{aligned}
& \mathbb{P}\left[\operatorname{LIC}\left(p^{*}\right)<\operatorname{LIC}\left(p_{0}\right)\right] \\
& =\mathbb{P}\left[\log \left(\frac{\widehat{\sigma}^{2}\left(p^{*}\right)}{\widehat{\sigma}^{2}\left(p_{0}\right)}\right)<\left(\frac{h(n \bar{T})}{n \bar{T}}+\frac{1}{\bar{T}^{2}}\right)\left(p_{0}-p^{*}\right)+\frac{1}{\bar{T}}\left(R\left(p_{0}\right)-R\left(p^{*}\right)\right)\right] .
\end{aligned}
$$

The left-hand-side of the inequality in A.8 is positive in the limit as $n, \bar{T} \rightarrow \infty$ because $\widehat{\sigma}^{2}\left(p_{0}\right)=\sigma^{2}+o_{p}(1)$ and $\widehat{\sigma}^{2}\left(p^{*}\right)=\sigma^{2}+A+o_{p}(1)$ for some $A>0$ (due to the underspecification) whenever $p^{*}<p_{0}$, as shown in Lemma 1 of Han et al. (2013). On the other hand, the right-hand-side of the inequality in A.8 converges to zero as $n, \bar{T} \rightarrow \infty$ since $0<\left(p_{0}-p^{*}\right)<\bar{p}<\infty,\left|R\left(p_{0}\right)-R\left(p^{*}\right)\right|<\infty$ from the invertibility in Assumption 4-(ii), and $h(n \bar{T}) / n \bar{T} \rightarrow 0$ as $n \bar{T} \rightarrow \infty$ by assumption. Therefore, $\limsup _{n, \bar{T} \rightarrow \infty} \mathbb{P}\left[L I C\left(p^{*}\right)<\right.$ $\left.L I C\left(p_{0}\right)\right] \leq \mathbb{P}\left[\lim \sup _{n, \bar{T} \rightarrow \infty}\left\{L I C\left(p^{*}\right)<L I C\left(p_{0}\right)\right\}\right]=\mathbb{P}[\varnothing]=0$.

For the case of over-selection, $p^{*}>p_{0}$, we note that $R\left(p^{*}\right)=\widehat{\omega}\left(p^{*}\right) / \widehat{\sigma}^{2}\left(p^{*}\right)$, where

$$
\widehat{\omega}\left(p^{*}\right)=\sum_{j=-m}^{m} K_{j} \widehat{\gamma}_{j}\left(p^{*}\right) \text { and } \widehat{\gamma}_{j}\left(p^{*}\right)=\frac{1}{n \bar{T}} \sum_{i=1}^{n} \sum_{t=\max \{\bar{p}+1, \bar{p}+j+1\}}^{\min \{T, T+j\}} \widetilde{\varepsilon}_{i, t}^{W}\left(p^{*}\right) \widetilde{\varepsilon}_{i, t-j}^{W}\left(p^{*}\right) \text {. }
$$

Under Assumption 4 and a proper choice of kernel (and truncation), $0<R(p)<\infty$. From Section 2.2, since $\widehat{\sigma}^{2}\left(p^{*}\right)$ and $\widehat{\omega}\left(p^{*}\right)$ are respectively bias-uncorrected estimators of the variance $\sigma^{2}\left(p^{*}\right)$ and the long-run variance $\omega\left(p^{*}\right)$ of $\varepsilon_{i, t}\left(p^{*}\right)$, we have $\widehat{\sigma}^{2}\left(p^{*}\right)=\sigma^{2}\left(p^{*}\right)+O_{p}(1 / \bar{T})$ and $\widehat{\omega}\left(p^{*}\right)=\omega\left(p^{*}\right)+O_{p}(1 / \bar{T})$. When $p^{*} \geq p_{0}$, furthermore, $\omega\left(p^{*}\right)$ becomes $\sigma^{2}\left(p^{*}\right)$ and thus $R\left(p^{*}\right)=1+O_{p}(1 / \bar{T})$ because $\varepsilon_{i, t}=\varepsilon_{i, t}\left(p_{0}\right)$ is White noise. It thus follows that for the over-selection case, $\left|R\left(p^{*}\right)-R\left(p_{0}\right)\right|$ is at most $O_{p}(1 / \bar{T})$. Now consider

$$
\begin{aligned}
& \mathbb{P}\left[\operatorname{LIC}\left(p^{*}\right)<\operatorname{LIC}\left(p_{0}\right)\right] \\
& =\mathbb{P}\left[n \bar{T}\left(\log \widehat{\sigma}^{2}\left(p^{*}\right)-\log \widehat{\sigma}^{2}\left(p_{0}\right)\right)+\left(\frac{n}{\bar{T}}\right)\left(p^{*}-p_{0}\right)+n\left(R\left(p^{*}\right)-R\left(p_{0}\right)\right)<h(n \bar{T})\left(p_{0}-p^{*}\right)\right] .
\end{aligned}
$$

As in the proof of Theorem 2 of Han et al. (2013) we have $n \bar{T}\left(\log \widehat{\sigma}^{2}\left(p^{*}\right)-\log \widehat{\sigma}^{2}\left(p_{0}\right)\right)=$ $O_{p}(1)$. Further, $n\left(R\left(p_{0}\right)-R\left(p^{*}\right)\right)=O_{p}(n / \bar{T})$ as described above. The left-hand-side of the inequality in the expression $(\mathrm{A} .9)$ is thus $O_{p}(1)$ for large $n$ and $T$ because it is assumed 
that $n / \bar{T} \rightarrow \kappa \in(0, \infty)$. On the other hand, the right-hand-side goes to negative infinity as $n \bar{T} \rightarrow \infty$ since $p_{0}-p^{*}<0$ and $h(n \bar{T}) \rightarrow \infty$. It follows that $\limsup _{n, \bar{T} \rightarrow \infty} \mathbb{P}\left[L I C\left(p^{*}\right)<\right.$ $\left.L I C\left(p_{0}\right)\right]=0$ for $p^{*}>p_{0}$.

Proof of Corollary 6 First note that in the case of over-selection (i.e., $p^{*}>p_{0}$ and $\left.p^{* *}>p_{0}\right)$, it holds that $p^{* *} \geq p^{*}$. This is because, from the discussion about $R(p)$ in the proof of Theorem 5, $R(p)>0$ is close to 1 for all $p \geq p_{0}$ if its $O_{p}(1 / \bar{T})$ bias is ignored. It thus follows that the penalty of $L I C(p),(p / n \bar{T})(h(n \bar{T}) / n \bar{T}+n / \bar{T})+R(p) / \bar{T}$, is generally non-decreasing in $p$ for $p \geq p_{0}$. Therefore, since the penalty of $I C(p)$ is strictly smaller than that of $L I C(p)$, we conclude that $p^{* *} \geq p^{*}$ for the over-selection case.

We now define

$$
\begin{aligned}
\Delta L I C & \equiv \operatorname{LIC}\left(p^{*}\right)-\operatorname{LIC}\left(p_{0}\right) \\
& =\log \left(\frac{\widehat{\sigma}^{2}\left(p^{*}\right)}{\widehat{\sigma}^{2}\left(p_{0}\right)}\right)+\frac{h(n \bar{T})}{n \bar{T}}\left(p^{*}-p_{0}\right)+\frac{1}{\bar{T}^{2}}\left(p^{*}-p_{0}\right)+\frac{1}{\bar{T}}\left(R\left(p^{*}\right)-R\left(p_{0}\right)\right)
\end{aligned}
$$

and

$$
\Delta I C \equiv I C\left(p^{* *}\right)-I C\left(p_{0}\right)=\log \left(\frac{\widehat{\sigma}^{2}\left(p^{* *}\right)}{\widehat{\sigma}^{2}\left(p_{0}\right)}\right)+\frac{h(n \bar{T})}{n \bar{T}}\left(p^{* *}-p_{0}\right) .
$$

Then, similar to the proof of Theorem 5, we write

$$
\begin{aligned}
& \mathbb{P}[\Delta L I C<\Delta I C] \\
= & \mathbb{P}\left[\log \left(\frac{\widehat{\sigma}^{2}\left(p^{*}\right)}{\widehat{\sigma}^{2}\left(p^{* *}\right)}\right)<\frac{h(n \bar{T})}{n \bar{T}}\left(p^{* *}-p^{*}\right)+\frac{1}{\bar{T}^{2}}\left(p_{0}-p^{*}\right)+\frac{1}{\bar{T}}\left(R\left(p_{0}\right)-R\left(p^{*}\right)\right)\right] .
\end{aligned}
$$

Since $p^{* *} \geq p^{*}$ in this case, the left-hand-side of the last inequality in A.10 is nonnegative for any $n$ and $\bar{T}$, whereas the right-hand-side goes to zero with $n, \bar{T} \rightarrow \infty$ as in A.8. Therefore, $\lim \sup _{n, \bar{T} \rightarrow \infty}\{\mathbb{P}[\Delta L I C<0]-\mathbb{P}[\Delta I C<0]\} \leq \lim \sup _{n, \bar{T} \rightarrow \infty} \mathbb{P}[\Delta L I C-\Delta I C<0] \leq$ $\mathbb{P}\left[\lim \sup _{n, \bar{T} \rightarrow \infty}\{\Delta L I C-\Delta I C<0\}\right]=\mathbb{P}[\varnothing]=0$. When $p^{*}>p_{0}$ and $p^{* *}>p_{0}$, as $\mathbb{P}[\Delta L I C<0]$ and $\mathbb{P}[\Delta I C<0]$ correspond to the over-selection probabilities of $L I C$ and $I C$, respectively, this result implies $\lim \sup _{n, \bar{T} \rightarrow \infty} \mathbb{P}\left(p^{*}>p_{0}\right) \leq \lim \sup _{n, \bar{T} \rightarrow \infty} \mathbb{P}\left(p^{* *}>p_{0}\right)$. 


\section{Acknowledgement}

The authors acknowledge helpful comments from Bruce Hansen, Chris Hansen, Yuichi Kitamura, Roger Moon, Peter Robinson and seminar participants at Yale, Columbia, Rochester, UVa, Boston College, Seoul National, MSU, Montreal, Pittsburgh, Alabama, Missouri, SUNYAlbany, SUNY-Binghampton, 2011 International Conference on Panel Data, 2011 CIREQ Conference, 2011 Midwest Econometrics Group, and NY Camp Econometrics IX. Lee thanks the Cowles Foundation for Research in Economics at Yale University, where he was a visiting fellow while working on this project. Phillips acknowledges support from the NSF under Grants No. SES 0956687 and SES 1258258. 


\section{References}

Akaike, H., 1973. Information theory and an extension of the maximum likelihood principle, in B.N. Petrov and B.F. Csaki (Eds.), 2nd International Symposium on Information Theory, 267-281, Budapest: Academia Kiado.

Akaike, H., 1974. A new look at the statistical model identification, IEEE Transactions on Automatic Control AC-19, 716-723.

Anderson, T. W. and C. Hsiao, 1981. Estimation of Dynamic Models with error components, Journal of the American Statistical Association 76, 598-606.

Andrews, D.W.K., 1994. Asymptotics for semi-parametric econometric models via stochastic equicontinuity, Econometrica 62, 43-72.

Arellano, M., and S. Bonhomme, 2009. Robust priors in nonlinear panel data models, Econometrica $77,489-536$.

Arellano, M. and J. Hahn, 2006. A likelihood-based approximate solution to the incidental parameter problem in dynamic nonlinear models with multiple effects, CEMFI Working Paper: No. 0613.

Arellano, M., and J. Hahn, 2007. Understanding bias in nonlinear panel models: Some recent developments, R. Blundell, W.K. Newey, and T. Persson eds., Advances in Economics and Econometrics: Theory and Applications, Ninth World Congress, Vol. III, Cambridge University Press.

Barndorff-Nielsen, O.E., 1983. On a formula for the distribution of the maximum likelihood estimator, Biometrika 70, 343-365.

Berger, J.O., J.K. Ghosh, and N. Mukhopadhyay, 2003. Approximations and consistency of the Bayes factors as model dimension grows, Journal of Statistical Planning and Inference $112,241-258$. 
Berger, J.O., B. Liseo, and R.L. Wolpert, 1999. Integrated likelihood methods for eliminating nuisance parameters, Statistical Science 14, 1-28.

Bester, C.A., and C. Hansen, 2009. A Penalty function approach to bias reduction in nonlinear panel models with fixed effects, Journal of Business and Economic Statistics 27, 131-148.

Bozdogan, H., 2000. Akaike's information criterion and recent developments in information complexity, Journal of Mathematical Psychology 44, 62-91.

Chakrabarti, A., and J.K. Ghosh, 2006. A generalization of BIC for the general exponential family, Journal of Statistical Planning and Inference 136, 2847-2872.

Claeskens, G. and N.L. Hjort, 2003. The focused information criterion, Journal of the American Statistical Association 98, 900-916.

Claeskens, G., and N.L. Hjort, 2008. Model Selection and Model Averaging, New York: Cambridge University Press.

Cox, D.R., and N. Reid, 1987. Parameter orthogonality and approximate conditional inference (with Discussion), Journal of the Royal Statistical Society B 49, 1-39.

DiCiccio, T.J., M.A. Martin, S.E. Stern, and G.A. Young, 1996. Information bias and adjusted profile likelihoods, Journal of the Royal Statistical Society B 58, 189-203.

Guyon, X., and J.-F. Yao, 1999. On the underfitting and overfitting sets of models chosen by order selection criteria, Journal of Multivariate Analysis 70, 221-249.

Hahn, J. and G. Kuersteiner, 2002. Asymptotically unbiased inference for a dynamic panel model with fixed effects, Econometrica 70, 1639-1657.

Hahn, J., and G. Kuersteiner, 2011. Bias reduction for dynamic nonlinear panel models with fixed effects, Econometric Theory 27, 1152-1191.

Hahn, J., and W. Newey, 2004. Jackknife and analytical bias reduction for nonlinear panel models, Econometrica 72, 1295-1319. 
Han, C., P.C.B. Phillips, and D. Sul, 2013. Lag length selection in panel autoregression, Working Paper, Yale University.

Heckman, J., and B.J. Singer, 1984. A Method for Minimizing the Impact of Distributional Assumptions in Econometric Models for Duration Data, Econometrica 52, 271-320.

Hodges, J.S. and D.J. Sargent, 2001. Counting degrees of freedom in hierarchical and other richly-parametrised models, Biometrika 88, 367-379.

Hsiao, C., 2003. Analysis of Panel Data, 2nd edition, Cambridge University Press.

Huber, P.J., 1981. Robust Statistics, New York: Wiley.

Kass, R. and A. Raftery, 1995. Bayes Factors, Journal of the American Statistical Association $90,773-795$.

Konishi, S. and G. Kitagawa, 1996. Generalized information criteria in model selection, Boimetrika 83, 875-890.

Lancaster, T., 2002. Orthogonal parameters and panel data, Review of Economic Studies 69, $647-666$.

Lee, Y., 2006. Nonparametric Approaches to Dynamic Panel Modelling and Bias Correction, Ph.D. dissertation, Yale University.

Lee, Y., 2012. Bias in dynamic panel models under time series misspecification, Journal of Econometrics 169, 54-60.

Lee, Y., 2014. Nonparametric estimation of dynamic panel models with fixed effects, Econometric Theory 30, 1315-1347.

Li, K.-C., 1987. Asymptotic optimality for $C_{p}, C_{L}$, cross-validation and generalized crossvalidation: Discrete Index Set, Annals of Statistics 15, 958-975.

McCullagh, P., and R. Tibshirani, 1990. A simple method for the adjustment of profile likelihoods, Journal of the Royal Statistical Society B 52, 325-344. 
Murphy, S.A., and A.W. van der Vaart, 2000. On Profile Likelihood. Journal of the American Statistical Association 95, 449-465.

Newey, W.K., 1994. The asymptotic variance of semiparametric estimators, Econometrica $62,1349-1382$.

Neyman, J. and E. Scott, 1948. Consistent estimates based on partially consistent observations, Econometrica 16, 1-32.

Phillips, P.C.B., 1980. The exact finite sample density of instrumental variable estimators in an equation with $n+1$ endogenous variables, Econometrica 48, 861-878.

Phillips, P.C.B., 1983. Marginal densities of instrumental variables estimators in the general single equation case, Advances in Econometrics 2, 1-24.

Phillips, P.C.B., 2014. Dynamic panel GMM with roots near unity, Working Paper, Yale University.

Phillips, P.C.B. and C. Han, 2014. The True Limit Distributions of the Anderson-Hsiao IV Estimators in Panel Autoregression, Working Paper, Yale University.

Rissanen, J., 1986. Stochastic Complexity and Modeling, Annals of Statistics 14, 1080-1100.

Sartori, N., 2003. Modified profile likelihoods in models with stratum nuisance parameters, Biometrika 90, 533-549.

Serfling, R., 1998. Approximation Theorems of Mathematical Statistics, Wiley.

Severini, T.A., 1998. An approximation to the modified profile likelihood function, Boimetrika $85,403-411$.

Severini, T.A., 2000. Likelihood Methods in Statistics, New York: Oxford University Press.

Severini, T.A. and W.H. Wong, 1992. Profile likelihood and conditionally parametric models, Annals of Statistics 20, 1768-1802. 
Shibata, R., 1980. Asymptotically efficient selection of the order of the model for estimating parameters of a linear process, Annals of Statistics 8, 147-164.

Stone, M., 1979. Comments on model selection criteria of Akaike and Schwartz, Journal of the Royal Statistical Society B 41, 276-278.

Tierney, L., R.E. Kass and J.B. Kadane, 1989. Fully exponential Laplace approximations to expectations and variances of nonpositive functions, Journal of the American Statistical Association 84, 710-716.

White, H., 1982. Maximum Likelihood Estimation of Misspecified Models, Econometrica 50, $1-25$.

Withers, C.S., 1983. Expansions for the distribution and quantiles of a regular functional of the empirical distribution with applications to nonparametric confidence intervals, Annals of Statistics 11, 577-587.

Yang, Y., 2005. Can the strengths of AIC and BIC be shared? A conflict between model identification and regression estimation, Biometrika 92, 937-950. 

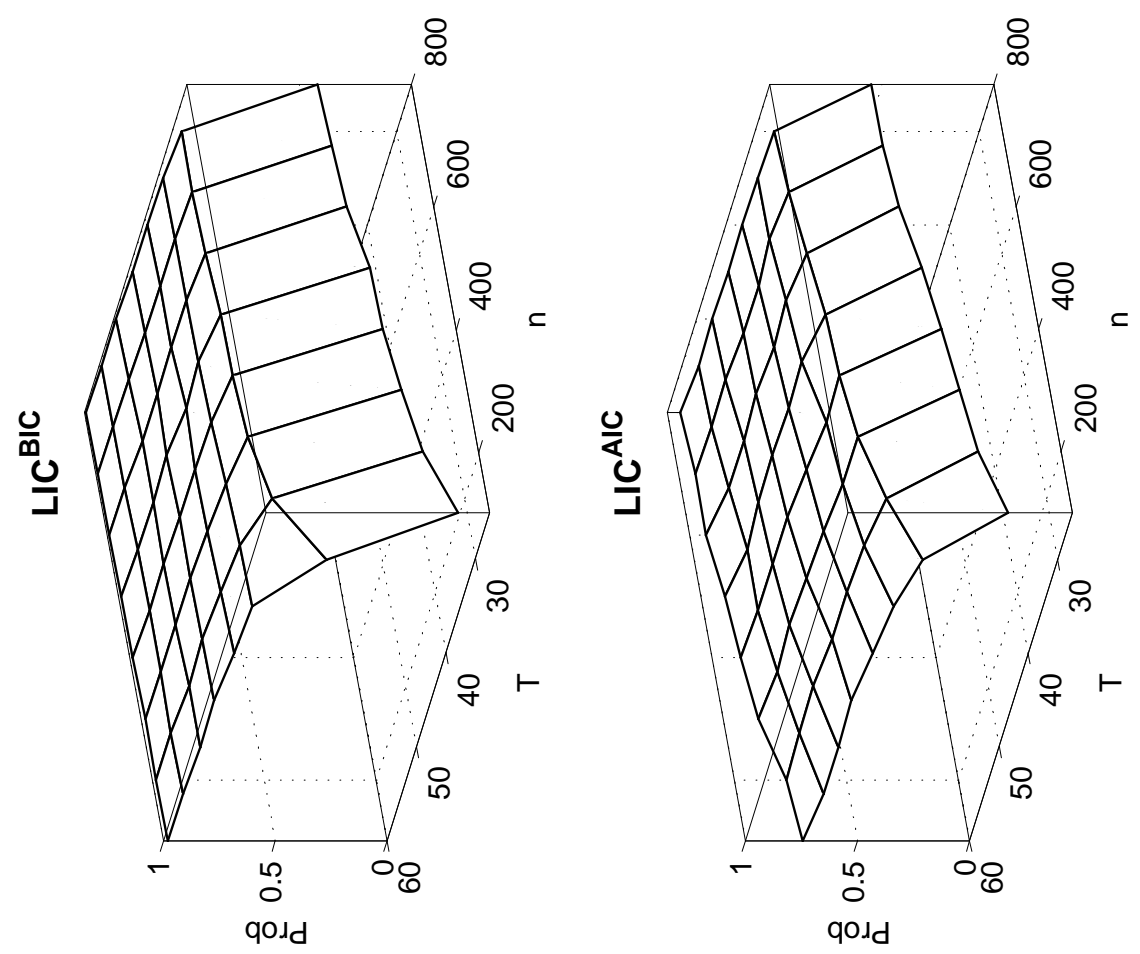

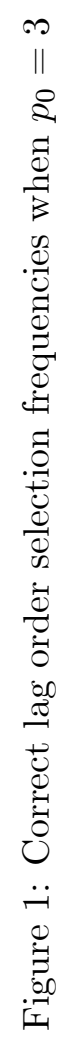
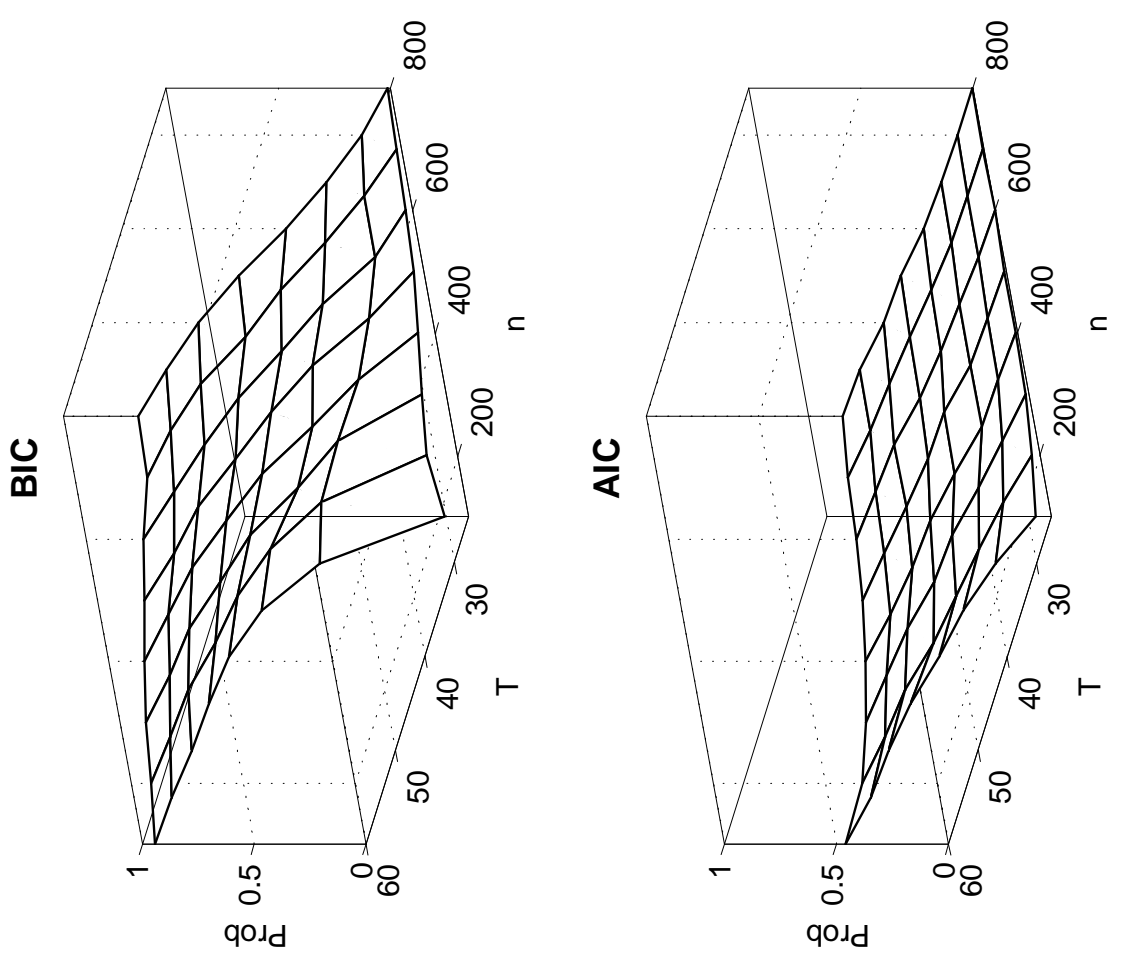

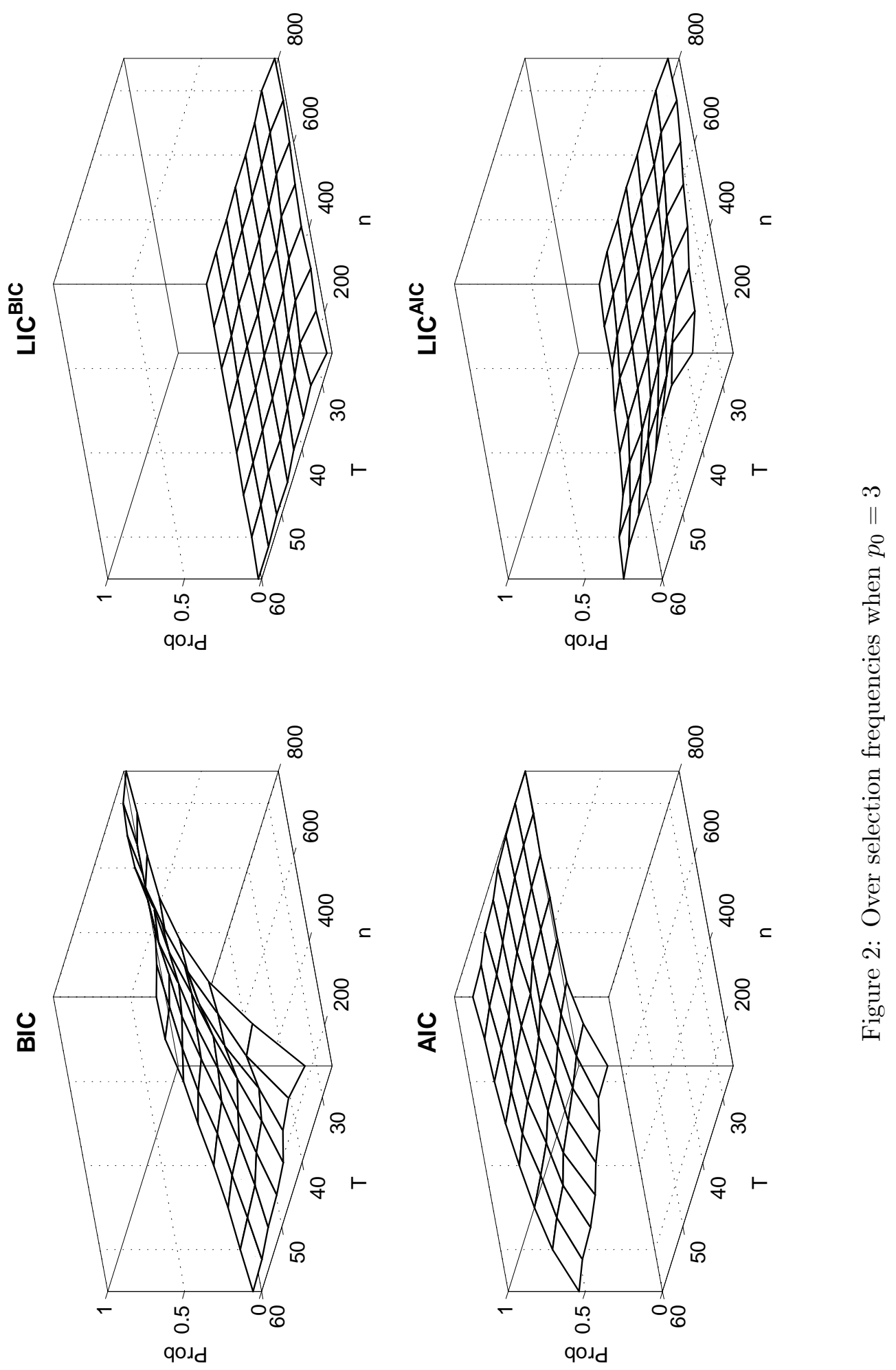

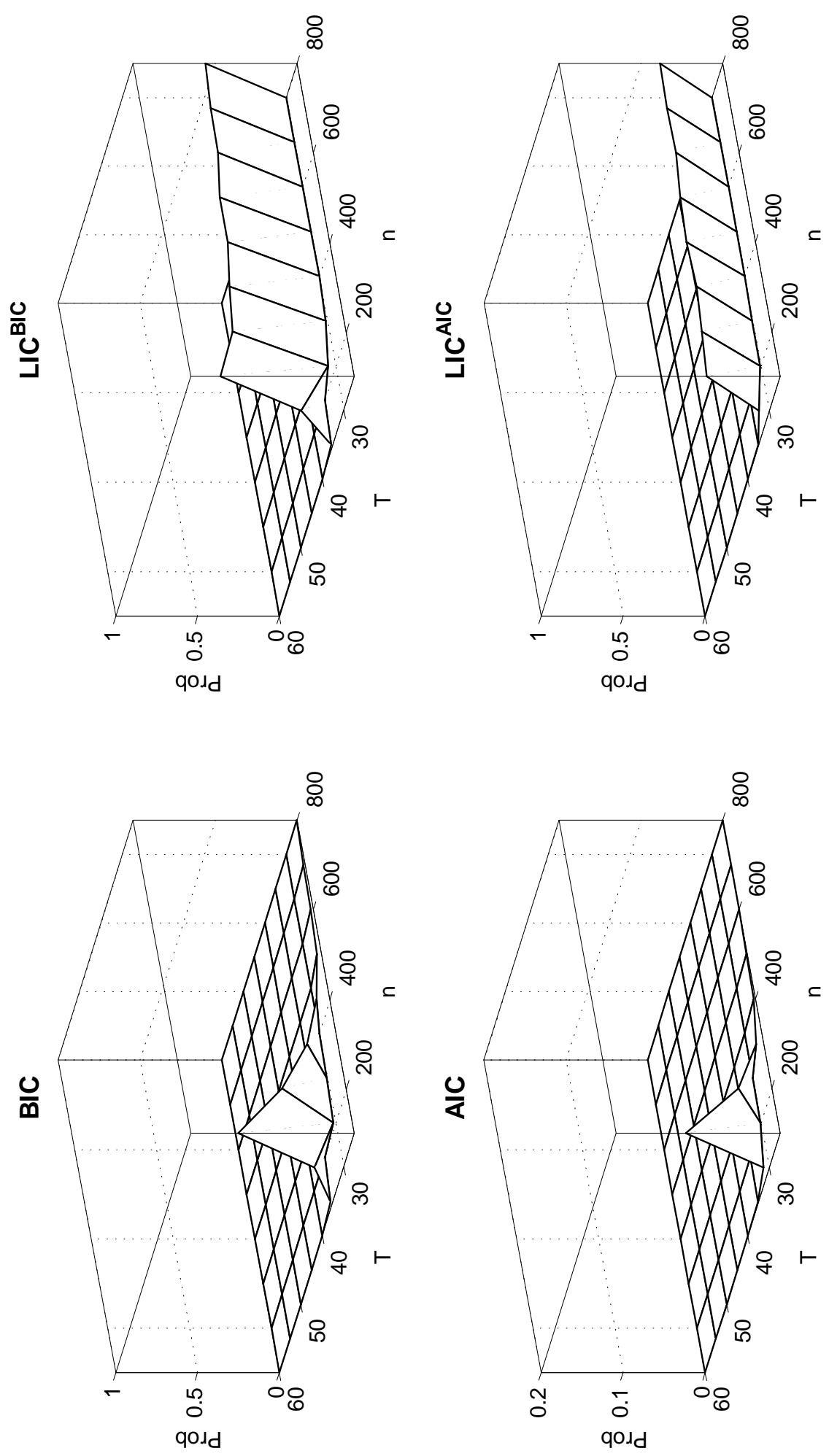

1 